\title{
Sesquiterpene emissions from vegetation: a review
}

\author{
T. R. Duhl ${ }^{1,2}$, D. Helmig ${ }^{1}$, and A. Guenther ${ }^{2}$ \\ ${ }^{1}$ Institute of Arctic and Alpine Research, University of Colorado, Boulder, CO 80309, USA \\ ${ }^{2}$ Atmospheric Chemistry Division, National Center for Atmospheric Research, Boulder, CO 80307, USA
}

Received: 13 September 2007 - Published in Biogeosciences Discuss.: 6 November 2007

Revised: 5 March 2008 - Accepted: 15 March 2008 - Published: 14 May 2008

\begin{abstract}
This literature review summarizes the environmental controls governing biogenic sesquiterpene (SQT) emissions and presents a compendium of numerous SQTemitting plant species as well as the quantities and ratios of SQT species they have been observed to emit. The results of many enclosure-based studies indicate that temporal SQT emission variations appear to be dominated mainly by ambient temperatures although other factors contribute (e.g., seasonal variations). This implies that SQT emissions have increased significance at certain times of the year, especially in late spring to mid-summer. The strong temperature dependency of SQT emissions also creates the distinct possibility of increasing SQT emissions in a warmer climate. Disturbances to vegetation (from herbivores and possibly violent weather events) are clearly also important in controlling short-term SQT emissions bursts, though the relative contribution of disturbance-induced emissions is not known. Based on the biogenic SQT emissions studies reviewed here, SQT emission rates among numerous species have been observed to cover a wide range of values, and exhibit substantial variability between individuals and across species, as well as at different environmental and phenological states. These emission rates span several orders of magnitude (10s-1000s of $n g \mathrm{~g}_{\mathrm{DW}}^{-1} \mathrm{~h}^{-1}$ ). Many of the higher rates were reported by early SQT studies, which may have included artificiallyelevated SQT emission rates due to higher-than-ambient enclosure temperatures and disturbances to enclosed vegetation prior to and during sample collection. When predicting landscape-level SQT fluxes, modelers must consider the numerous sources of variability driving observed SQT emissions. Characterizations of landscape and global SQT fluxes are highly uncertain given differences and uncertainties in experimental protocols and measurements, the high variabil-
\end{abstract}

Correspondence to: D. Helmig (detlev.helmig@ colorado.edu) ity in observed emission rates from different species, the selection of species that have been studied so far, and ambiguities regarding controls over emissions. This underscores the need for standardized experimental protocols, better characterization of disturbance-induced emissions, screening of dominant plant species, and the collection of multiple replicates from several individuals within a given species or genus as well as a better understanding of seasonal dependencies of SQT emissions in order to improve the representation of SQT emission rates.

\section{Introduction}

Vegetation emits a vast array and substantial quantities of biogenic volatile organic compounds (BVOC), including terpenoid BVOC (isoprene, monoterpenes, sesquiterpenes) and oxygenated hydrocarbons (alcohols, aldehydes and ketones) (Kesselmeier and Staudt, 1999). BVOC play a major role in air quality, secondary organic aerosol (SOA) formation, carbon sequestration, and biospheric interactions (Atkinson and Arey, 2003). Recent studies indicate that BVOC fluxes recycle a considerable amount of photosynthetically fixed carbon to the atmosphere (Kesselmeier et al., 2002). These findings suggest that BVOC emissions estimates must be included in global carbon budget calculations and air-quality assessments. As the emissions of many BVOC are highly temperature-dependant (Guenther et al., 1993; Harley et al., 1996; Westberg et al. 2001), BVOC fluxes will likely increase during this century as a result of predicted global temperature increases (Kulmala et al., 2005; Lathière et al., 2006).

Among numerous identified BVOC, sesquiterpenes (SQT) have been among the least studied, largely because SQT analysis is challenging given the high reactivity and relatively low vapor pressure of SQT. If specific sampling protocols are not adhered to, SQT can either be lost (causing

Published by Copernicus Publications on behalf of the European Geosciences Union. 
even high SQT fluxes to be overlooked) or artificially enhanced (due to disturbances). However, several recent research projects and reviews have specifically addressed the analytical requirements for quantitative SQT emissions measurements and provide a framework for SQT measurements from enclosures and in ambient air (Komenda et al., 2001; Merfort, 2002; Helmig et al., 2003, 2004; Pollmann et al., 2005; Tholl et al., 2006).

SQT emissions are known to vary considerably between vegetation species (Arey et al., 1995; König et al., 1995; Ciccioli et al., 1999; Helmig et al., 1999, 2007; Geron et al., 2006). There are numerous other drivers controlling SQT emissions, including both biotic and abiotic factors. This literature review is presented with the objective of summarizing the current state of knowledge regarding the environmental controls governing biogenic SQT emissions. Additionally, this review contains a compendium of SQT-emitting plant species as well as the quantities and ratios of SQT these plants have been found to emit. Following a brief introduction (Sect. 1), Sect. 2 summarizes the controls (both abiotic and biotic) over biogenic SQT emissions as elucidated by numerous enclosure-based studies, sorted by individual factors (light, disturbance, etc.). Section 3 presents two tables. The first outlines some of the known SQT-emitting plant species and the quantities of SQT they have been observed to emit, while the second summarizes the ratios of individual SQT chemical species reported in various studies. Section 4 offers a brief discussion of the findings reported here and some concluding remarks.

\section{Environmental controls over biogenic sesquiterpene emissions}

\subsection{Abiotic controls}

\subsubsection{Temperature}

All of the studies that have examined the temperature dependencies of SQT emissions have found that emissions are highly correlated with temperature. For example, BVOC emissions from several Citrus varieties were found to be dominated by $\beta$-Caryophyllene ( $\beta$-Car), with temperature being the main environmental control (Ciccioli et al., 1999). The investigators plotted $\beta$-Car emission data as function of leaf temperature relative to $30^{\circ} \mathrm{C}$ and obtained an exponential curve of normalized emissions. Based on emissions measurements from the branch of a young orange tree, Hansen and Seufert (1999) constructed a temperature-versusemissions curve for $\beta$-Car, which showed a 5.6-fold increase in emissions for air temperature increases of $10^{\circ} \mathrm{C}$. The authors suggested that perhaps there is a threshold temperature below which $\beta$-Car emissions cannot occur. Temperature experiments performed during a study of young corn plants showed that the proportion of $\beta$-Car as a percentage of to- tal BVOC emissions was highest at $37^{\circ} \mathrm{C}$ (Gouinguené and Turlings, 2002). Emissions of MT and SQT from Loblolly Pine trees exhibited a strong dependence on temperature, as well as a strong diurnal SQT emission pattern with significant increases through the morning, a peak in the early afternoon, and subsequent declines into the evening (Helmig et al., 2006). SQT emissions were found to exhibit a stronger temperature dependence than MT, indicating that SQT fluxes have increased significance at high ambient temperatures $\left(>30^{\circ} \mathrm{C}\right)$.

Comparisons between the various published SQT emissions studies are sometimes hindered by a lack of standardized emission rate (ER) reporting practices. Basal emission rates (BERs) are ERs that have been normalized to a standard temperature (usually $30^{\circ} \mathrm{C}$ ), and are useful for making comparisons among studies. Some investigators report ERs which have been normalized according to the temperaturedependant algorithms developed by Guenther et al. (1993) for monoterpenes, or through other formulations such as the Tingey algorithm (e.g., Ciccioli et al., 1999). There have also been numerous studies in which emissions are reported as observed under various temperature and light intensities. The selection of an ER reporting approach should depend on whether emissions are obtained using relatively narrow light and temperature ranges or observed under varying temperatures and light intensities. SQT ERs are often standardized using the following exponential relationship between ER and leaf temperature (Guenther et al., 1993):

$\mathrm{ER}=E(s) \exp [\beta(T-T(s))]$

Here, ER is the emission rate (generally expressed in $\mu \mathrm{g} \mathrm{g}_{\mathrm{DW}}^{-1} \mathrm{~h}^{-1}$, where $\mathrm{DW}$ is leaf dry weight in $\mathrm{g}$ ) observed at temperature $T, E(s)$ is the emission rate at standard temperature $(T(s))$, and $\beta$ is an empirically-derived coefficient based upon the best-fit curve obtained from plotting ln ER versus $T$. While some investigators normalize SQT emissions using $\beta$-factors obtained from MT emission rate studies, others derive this value empirically using SQT emissions data. Table 1 contains $\beta$-factors reported in various published SQTrelated studies, as well as which compound(s) were considered in the empirical determination of this parameter and the range of temperatures used to derive this value experimentally. Calculated $\beta$-factors ranged from 0.05 to $0.29 \mathrm{~K}^{-1}$ in a single study that characterized a wide range of pine species (Helmig et al., 2007). All other studies report values that fall within this range.

\subsubsection{Light}

Among the studies that have examined the light dependency of SQT emissions, there have been mixed findings, with evidence that some emissions are solely temperature-controlled while others are also affected by light. However, each of the studies that did not observe light dependencies in SQT emissions were conducted in field conditions, under ambient light 
Table 1. $\beta$-Factors, chemical species, and temperature range data for $\beta$-values that were determined empirically.

\begin{tabular}{|c|c|c|c|}
\hline Compound & $\beta$-Factor $\left(\mathrm{K}^{-1}\right)$ & $\begin{array}{l}\text { Measurement } \\
\text { temp. range, }{ }^{\circ} \mathrm{C}\end{array}$ & Reference \\
\hline Total sesquiterpenes & $\begin{array}{l}0.05-0.29 \\
\text { average: } 0.17\end{array}$ & $7 \rightarrow 43$ & Helmig et al. (2007) \\
\hline Total sesquiterpenes & $0.056-0.193$ (used 0.143 ) & $5 \rightarrow 40$ & Ruuskanen et al. (2007) \\
\hline$\beta$-caryophyllene & $0.175,0.201$ (used 0.19 ) & Not given & Hakola et al. (2006) \\
\hline 4 different SQT & $\begin{array}{l}0.12-0.18 \\
\text { average: } 0.15\end{array}$ & $4 \rightarrow 38$ & Helmig et al. (2006) \\
\hline$\beta$-caryophyllene & $\begin{array}{l}0.15 \text { (May), } 0.12 \text { (June), } \\
0.16 \text { (autumn), } 0.19 \text { (early } \\
\text { summer) }\end{array}$ & $-5 \rightarrow 32$ & Tarvainen et al. (2005) \\
\hline Total sesquiterpenes & $\begin{array}{l}0.14-0.22 \\
\text { average: } 0.19\end{array}$ & Not given & Hakola et al. (2001) \\
\hline$\beta$-caryophyllene & 0.17 & $24 \rightarrow 31$ & Hansen and Seufert (1999) \\
\hline MT and SQT & 0.09 & $12 \rightarrow 39$ & Arey et al. $(1995)^{*}$ \\
\hline
\end{tabular}

* In this study, the $\beta$-factor was empirically determined using data collected from a single Monterey Pine tree, which yielded a $\beta$-value of $0.085 \pm 0.014 \mathrm{~K}^{-1}$. Subsequently, a $\beta$-factor of $0.09 \mathrm{~K}^{-1}$ was used to normalize emissions of total MT+SQT for all MT/SQT-emitting species that were screened.

and temperature regimes. As noted by Hakola et al. (2006), it can be difficult to discern light dependencies from emissions data collected during field experiments, where measurements may be performed under light-saturated conditions. Much of the literature suggests that dependencies on temperature are much stronger than those for light (e.g., Helmig, 2006).

$\beta$-Car emissions from sunflower were observed to be both light- and temperature-dependent (Schuh et al., 1997). By varying light intensity, but not temperature, the investigators constructed a light-dependent curve for $\beta$-Car emissions. At a constant leaf temperature of $23^{\circ} \mathrm{C}, \beta$-Car emission was invariable at PPFDs between 0 and $275 \mu \mathrm{mol} \mathrm{m}^{-2} \mathrm{~s}^{-1}$; but emission roughly doubled each time PPFD was increased by $275 \mu \mathrm{mol} \mathrm{m}^{-2} \mathrm{~s}^{-1}$ up to a threshold of $825 \mu \mathrm{mol} \mathrm{m}^{-2} \mathrm{~s}^{-1}$. When PPFD was adjusted from 825 to $1100 \mu \mathrm{mol} \mathrm{m}^{-2} \mathrm{~s}^{-1}$, the emission of $\beta$-Car only increased by $\sim 50 \%$. In this study, emissions were detected at night (at low levels), and it was suggested that these could be attributed to simple diffusion from storage pools (a temperature-dependent process). The investigators ruled out stomatal control as a factor affecting the BVOC flux after experimenting with varying degrees of stomatal conductance. The authors concluded that BVOC emission rates from sunflower depend both on biosynthesis rates as well as diffusion out of pools. In Downy Birch, Hakola et al. (2001) found that SQT emissions continued after light was prevented from reaching branches.

$\beta$-Car emissions from the branch of a young orange tree were not detected in the dark even when temperatures were comparable to those under lighted conditions (Hansen and Seufert, 1999). In a later study, Hansen and Seufert (2003) analyzed the $\beta$-Car emissions data from Ciccioli et al. (1999) using two algorithms to determine whether observed emis- sion rates were better modeled using a temperature-only or a light- and temperature-based algorithm. The algorithm that assumed both light and temperature dependencies produced modeled data points that more closely matched observations than values obtained by assuming emissions were solely temperature-dependent. However, Ciccioli et al. (1999) reported that emission rates were similar for branches growing in full versus half-shaded areas, and concluded that $\beta$-Car emissions were unaffected by varying stomatal conductance.

Young corn plants were found to emit several SQT chemical species which responded differently to changing PPFDs (Gouinguené and Turlings, 2002). Under constant chamber temperatures $\beta$-Car emissions were positively correlated with light intensity, $\alpha$-farnesene and $\beta$-bisabolene were negatively correlated, and other SQT species were unaffected by changing light levels. Under increasing light-intensity conditions, significant increases in total induced BVOC emissions occurred, while relative contributions from $\alpha$-farnesene and $\beta$-bisabolene to total emissions declined. $\beta$-Car increased from about $1.3 \%$ to $2.8 \%$ of total BVOC emissions when illuminance changed from $0-5000 \mathrm{~lm} \mathrm{~m}^{-2}$. The investigators noted that emissions responded sharply to changes in lightdark cycles, an indication that circadian rhythms were not controlling these releases (see Sect. 2.1.5).

Tomato shoots exposed to perpetual light exhibited continuous emission of $\alpha$-copaene (Maes and Debergh, 2003). During this experiment, the emission of $\beta$-Car declined, while levels of $\alpha$-copaene increased until they exceeded those of $\beta$-Car, a behavior not observed under unstressed (i.e., normal light-dark phase) conditions. When the emission profile of $\alpha$-copaene (for an unstressed tomato shoot) was examined, a clear diurnal emission pattern was evident, 
with an increase during the day and a decline at night. The investigators suggested that $\alpha$-copaene requires light for its biosynthesis and/or emission. During the continuous light experiments, temperature inside the experimental room was maintained at $27^{\circ} \mathrm{C}$, and PPFD was constant at $70 \mu \mathrm{mol} \mathrm{m}^{-2} \mathrm{~s}^{-1}$. A biotic stressor (caterpillar herbivory) was also applied to tomato shoots, and elevated levels of SQT were observed (see Sect. 2.2.3). In Scots Pine, $\beta$-Car was the dominant SQT, emitted at levels representing $2-5 \%$ (southern Finland) and 40\% (northern Finland) of measured springtime MT emissions (Tarvainen et al., 2005). SQT emissions were not found to be light-dependent, and appeared to vary seasonally (see Sect. 2.1.4).

Though Helmig et al. (2006) concluded that light influenced SQT emissions from Loblolly Pine, the variables of light and temperature were difficult to separate given the ambient conditions observed during the course of their study. Despite this, the authors found the best fit of modeled data to observed values when SQT emissions were assumed to be $20 \%$ temperature-only and $80 \%$ light- and temperaturedependent. The BVOC emissions of 40 year-old Scots Pine trees were studied under varying temperatures and light intensities and over changing seasons (Holzke et al., 2006). Standardized emissions algorithms (after Guenther et al., 1993) were computed to evaluate emission rate dependencies on light and temperature. SQT accounted for up to $6 \%$ of total measured BVOC emissions, but had a strong seasonal variation (see Sect. 2.1.4). Despite the fact that emissions were not detected at night, the effects of light were not found to be significant.

\subsubsection{Diurnal emission patterns}

All studies involving volatile collection at multiple times during the day report diurnal rhythmicity in SQT emissions. When Black Sage (Salvia mellifera) plants were sampled throughout the day, total emission rates (and SQT emission rates in particular) were higher in samples obtained earlier in the day (Arey et al. 1995). Since there was a relatively narrow temperature range recorded during the course of this study, one explanation given for the daily decline in SQT emissions is that there are limited storage pools which are depleted each day, a finding that parallels previous observations made on MT emission rates for Black Sage (Dement et al., 1975). In the Arey et al. study, ten native plant species representing significant sources of biomass within the South Coast Air Basin of California were screened for BVOC emissions, and three of these species (Black Sage, California Sagebrush, and Greenbark) emitted SQT.

Volatile emissions from developing rose flowers obeyed a diurnal, circadian pattern when the effects of altered lightdark regimes were tested (Helsper et al., 1998). During a 12-h photoperiod experiment, SQT emissions tended to peak $6-9 \mathrm{~h}$ into the photoperiod, but never dropped off completely even in dark periods. $\beta$-Car was not emitted during a 5-day period of extended darkness, though $\beta$-cubebene emissions were observed to increase during this time. When flowers were exposed to a 12 -h photoperiod, followed by either continuous light or continuous darkness, emission rhythmicity in both cases was found to continue for two or three cycles and then gradually decrease. The authors postulated that observed rhythmicity has a biochemical, and not a physical (e.g., petal closure) basis, and that emission of $\beta$-Car is lightdependent. In a study of snapdragon flowers, rhythmicity of the nonmevalonate (i.e., MEP) pathway was found to affect nerolidol biosynthesis and emission more directly than light, although it was also noted that in these flowers both processes occur in non-photosynthetic cells (Dudareva et al., 2005).

$\beta$-Car emissions from various Citrus varieties increased during the morning and generally peaked around noon (Ciccioli et al., 1999). Stomatal closure was not found to affect emissions, as $\beta$-Car (and several monoterpenes) was detected at night, and no correlation was observed between BVOC emissions and uptake of $\mathrm{CO}_{2}$.

Major compounds released by both young and mature potato plants during morning, noon, and afternoon hours were quantified to characterize diurnal rythmicity of emissions and emission variability among individual plants (Agelopoulos et al., 2000). Investigators also looked for a relationship between foliage weight and emissions for both young and mature plants. Potatos were found to emit predominantly SQT, and the composition of emitted compounds was constant during the photoperiod and between young and mature plants. Quantities of emitted SQT increased steadily throughout the day and peaked in the afternoon for both young and mature plants, except for the release of an unknown compound (from young plants) which was emitted at relatively constant amounts all day (see Sect. 2.2.1).

Herbivore-induced nighttime BVOC emissions of tobacco plants were studied based on the hypothesis that selected species of ovipositing female moths are repelled by herbivore-infested plants which emit these volatile blends as a result of herbivory (see Sect. 2.2.3) (De Moraes et al., 2001). Several non-SQT compounds which were not present in the daytime samples were detected at night. SQT were emitted both during the day and night, but emission rates were 1.2-3 times higher in the daytime. Release of germacrene-D from forest tent caterpillar-infested poplar leaves exhibited a diurnal rythmicity with emissions maxima occurring during the photoperiod (06:00-18:00 h) and extremely low (often undetectable) quantities detected during dark periods (Arimura et al., 2004). In an ambient study of Siberian Larch, diurnal fluctuations of SQT and MT emissions were attributed to temperature variations (Ruuskanen et al., 2007). 


\subsubsection{Seasonality}

Some studies indicate that seasonality can be as important as temperature in exerting control over biogenic SQT release, while others have determined that emissions can be adequately described by temperature alone. When Black Sage plants were monitored in the field (between mid-February and December), changes in the ratios of SQT emissions (as proportions of total emitted BVOC) comprised the largest change in emission profiles (Arey et al., 1995). The investigators suggested that perhaps the phenological state of the Black Sage controls SQT production, as this was observed to be higher prior to the peak blooming of the plants in May, or that the high SQT production was a response to aphid infestation (see Sect. 2.2.3). The authors did not believe that enhanced SQT ratios were caused by ambient temperatures, since proportions were low in August (when temperatures were high) and high in December (when night temperatures were low). The authors concluded that overall, there was not a significant seasonal dependence of BVOC emissions, but that disturbances to the plants may have exerted the most influence on total observed emission variability.

BVOC emissions within a Citrus orchard in Spain were measured during April-May, July, and again in October. $\beta$ Car was the dominant BVOC emitted by 6 different Citrus varieties during July sampling, comprising $50-70 \%$ of all detected hydrocarbons, but total emission rates varied by up to one order of magnitude between cultivars (Ciccioli et al., 1999). Emissions of all BVOC were negligible during October. The authors noted that SQT are thought to be synthesized outside of chloroplasts and stored in pools. For samples collected from April to May, when temperatures were $5-10^{\circ} \mathrm{C}$ lower than average July temperatures, a dramatic reduction in $\beta$-Car emissions was observed.

Several species of deciduous trees exhibited significant changes in BVOC emissions between June and August (Zhang et al., 1999). Since light and temperature regimes were nearly the same during these two months, the authors attributed these changes to leaf maturation. Higher emissions were observed at higher temperatures, however. For $\beta$-Car, emissions rose sharply between 16 and $24^{\circ} \mathrm{C}$, then plateaued. Temperatures recorded for June sampling were similar to those recorded during August. Downy Birch emitted by far the highest quantities and largest number of SQT and oxygenated SQT. Among these, $\beta$-Car, caryophyllene oxide, and $\alpha$-copaene were emitted in the greatest quantities. In June, bourbonene, $\alpha$-copaene, and $\beta$-Car were among the major compounds detected from European Birch. Trembling Aspen and Black Elder emitted fewer SQT than the birch species. For August samples from all four species, fewer SQT were detected, and at lower levels than those recorded in June. An exception was muurolene, which was released by European Birch at higher levels in August than in June.

Measurements from Scots Pine were taken from early spring through autumn in southern and northern Finland
(Tarvainen et al., 2005). $\beta$-Car emissions exhibited significant seasonal variability, with maximum emissions observed during summer months. A similar trend was observed for this species by Hakola et al. (2006), who found that the highest emissions potentials for $\beta$-Car and other SQT (after being normalized to $30^{\circ} \mathrm{C}$ ) occurred in July.

When emissions from two Silver Birch trees were measured in July and August, July emissions were SQTdominated, with SQT comprising 39-71\% of total BVOC, while August samples were MT-dominated, with SQT making up 16-36\% of total emissions (Vuorinen et al., 2005; also see Sect. 2.1.5).

Standardized SQT ERs varied seasonally in Siberian Larch, with $\alpha$-farnesene dominating the $\sim 12 \%$ contribution of SQT to total summertime BVOC emissions. In the fall, SQT comprised only $\sim 3 \%$ of emitted BVOC, and the percent contribution of $\alpha$-farnesene to total SQT also fell significantly (Ruuskanen et al., 2007).

The fractional contribution of SQT to total BVOC emissions from Scots Pine displayed a strong seasonal variation, with much higher percentages of SQT (up to $\sim 6 \%$ of total terpene emissions) found in the spring/early summer than were measured in August-October (Holzke et al., 2006). The authors noted that this strong seasonal SQT variability could create unique chemical regimes during different seasons. Investigators also observed that light and temperature variability alone did not explain the observed trends and that both endogenous (e.g., developmental stage of branch) and exogenous (e.g., temperature) factors were likely responsible for the variability observed during the course of the year. The authors suggested the development of emissions algorithms incorporating both physiological and ontogenic parameters in order to better predict BVOC fluxes.

\subsubsection{Other abiotic factors}

Light and temperature are not the only abiotic drivers of variability in SQT emissions. Soil moisture and air humidity, plant water stress, and fertilization levels can also contribute to changes in SQT fluxes, while trace gas concentrations $\left(\mathrm{CO}_{2}, \mathrm{O}_{3}\right)$ appear to have less impact on observed variability. The effects of water stress on terpenoid emissions from the branch of a young orange tree were investigated by Hansen and Seufert (1999). Samples were collected at roughly the same time each day. $\beta$-Car accounted for a significant portion $(40-45 \%)$ of total emissions. Severe drought reduced $\beta$-Car emissions to $<6 \%$ of pre-drought levels, but emissions were unaffected by mild drought conditions. Similarly, Ormeño et al. (2007b) observed reduced SQT emissions following drought stress in Rosemary, Kermes Oak, Aleppo Pine, and Rock Rose.

Induced BVOC emissions from young corn plants were studied under varying soil moisture, air humidity, temperature, light intensity and rhythm, and at different fertilization rates (Gouinguené and Turlings, 2002). All factors were 
tested independently of each other. Emissions were induced after application of caterpillar oral secretion, which was either directly injected into stems or applied to leaves that had been mechanically damaged. Generally, the plants did not emit significant levels of BVOC when they were undamaged, irrespective of the abiotic stressor applied. In the presence of herbivore wounding, however, the plants emitted several SQT and the quantities and ratios of these compounds were affected by all abiotic stressors. The investigators noted a negative relationship between soil moisture and induced emissions. However, two SQT were shown to increase in terms of their fractional contribution to emissions as soil moisture increased. Induced emissions were highest at relative air humidities between $45 \%$ and $65 \%$, and no humidity-related effect was observed on the composition of odor blends. Fertilization rate negatively affected induced emissions, even when these were normalized to plant biomass. Percentages of the SQT $\alpha$-bergamotene and (E)- $\beta$ farnesene did not change when fertilization rates were varied, however. Certain SQT compounds appeared to be less sensitive to changes in various abiotic factors than other compounds.

SQT emissions from Rosemary, Aleppo Pine, and Rock Rose did not vary according to soil nutrient content when emissions from these species were studied across siliceous and calcareous soils (Ormeño et al., 2007a). Exceptions are the standardized emissions of $\alpha$-humulene from Aleppo Pine, which was higher in specimens growing in calcareous soils, and of $\alpha$-humulene and $\beta$-bourbonene from Rock Rose, which were higher in specimens growing in siliceous soils. Despite the general lack of correlation between SQT emissions and soil type, SQT emitted from both Rosemary and Aleppo Pine comprised a higher percentage of total BVOC emissions in plants growing in siliceous soils.

The effects of elevated $(\sim 2 \times$ ambient $) \mathrm{CO}_{2}$ on induced emissions of cabbages (which included the SQT $\alpha$ farnesene) subjected to insect herbivory were examined by Vuorinen et al. (2004). The results suggested no statistically significant effect on induced SQT emissions from plants grown under these conditions. In another study, BVOC emissions from two Silver Birch clones grown under ambient and elevated $\mathrm{CO}_{2}$ and at varying $\mathrm{O}_{3}$ concentrations were measured (Vuorinen et al., 2005). The variables were tested separately as well as in concert, and results indicated that elevated atmospheric concentrations of $\mathrm{CO}_{2}$ and $\mathrm{O}_{3}$ do not significantly affect emissions of MT or SQT from this species.

\subsection{Biotic controls}

\subsubsection{Plant developmental stage}

Numerous authors have noted that the release of SQT appears to vary not only according to light and temperature intensity, time of day, and season, but is also determined by the phenological state of a plant (e.g., leaf age, blossoming). During
BVOC sampling of several Citrus cultivars, multiple cuvettes were placed within a given tree in order to ascertain the effects on emissions from immature fruits, sun/shade regimes, and the presence of flowers (Ciccioli et al., 1999). The presence of semi-mature fruits within some enclosures did not have a significant effect on overall emissions. During springtime (April-May) sampling, many of the branches contained blossoms, and total emissions from flowering branches were approximately an order of magnitude higher than emissions from non-flowering branches. Furthermore, these emissions were not dominated by $\beta$-Car, but instead by MT. Hansen and Seufert (1999) found that emissions from the branch of an orange tree that contained blossoms had terpene emissions almost eight times higher than samples collected after the removal of blossoms. However, SQT accounted for a similar percentage of the total terpene emissions from the branch irrespective of whether or not flowers were present.

Ratios of total SQT emissions to $\beta$-farnesene were relatively stable in both young and mature potato plants, though variability in quantities of SQT emissions was highest (varying by up to an order of magnitude) among young plants (Agelopoulos et al., 2000). Mature plants exhibited a greater correlation between foliage weight and emissions than young plants. The BVOC emission rates of young trees from two birch species were quantified over the course of a growing season in experiments by Hakola et al. (2001). One species, Silver Birch (B. pendula), did not emit SQT, while Downy Birch (B. pubescens) did. Three years after their first measurements, the authors again studied emissions from $B$. pubescens, this time using older trees. Mature $B$. pubescens emitted higher proportions of SQT than younger trees, and at greater quantities. Emissions data following leaf initiation in the spring were analyzed as a function of Effective Temperature Sum (ETS) and presented in terms of ranges of degree days (d.d.). SQT emissions potentials for B. pubescens appear to be highest between 400-800 d.d.

\subsubsection{Disturbance}

Disturbances to vegetation during enclosure set-up have been observed to cause significant bursts in SQT emissions. Arey et al. (1995) noted that when Black Sage plants were not handled carefully during enclosure set-up, disturbance-related emissions bursts occurred which were roughly an order of magnitude higher (up to $205 \mu \mathrm{g} \mathrm{g}_{\mathrm{DW}}^{-1} \mathrm{~h}^{-1}$ ) than reported averages. However, ratios of individual compounds did not change much. Hakola et al. (2001) also observed that rough handling caused temporary but significant increases in emissions from birch trees.

The potential for artificially increasing emission rates during the setup of the enclosure system was not realized until relatively recently. As researchers began paying more attention to this effect, enclosure techniques have been improved. Researchers will now typically wait a considerable time $(\sim 12-24 \mathrm{~h})$ after installation of the enclosure and prior 
Table 2. Compendium of studies of biogenic SQT emission, plant species used in each study, the quantities and qualities of SQT emitted, and whether or not reported ERs can be considered quantitative.

\begin{tabular}{|c|c|c|c|c|c|c|c|c|}
\hline \multirow[b]{2}{*}{ Reference } & \multirow[b]{2}{*}{ Plant Species } & \multirow[b]{2}{*}{ Chemical Species } & \multicolumn{4}{|c|}{ Emission Rates (ERs) } & \multirow[b]{2}{*}{ 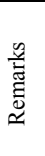 } & \multirow[b]{2}{*}{ 苞 } \\
\hline & & & ng $\mathrm{g}_{\mathrm{DW}}{ }^{-1}$ hour $^{-1}$ & $\begin{array}{l}\mathrm{ng} \mathrm{hr}^{-1} \\
\text { biomass } \\
\text { not } \\
\text { reported }\end{array}$ & $\mathrm{ng} \mathrm{m} \mathrm{m}^{-2} \mathrm{hr}^{-1}$ & $\begin{array}{l}\text { SQT \% of } \\
\text { total } \\
\text { BVOC }\end{array}$ & & \\
\hline \multirow{6}{*}{$\begin{array}{l}\text { Agelopoulos et } \\
\text { al., } 2000\end{array}$} & \multirow{6}{*}{$\begin{array}{l}\text { Potato plants; } \\
\text { Solanum } \\
\text { tuberosum L. cv. } \\
\text { Desireé }\end{array}$} & $\beta-$ Car & & $609-1276$ & & $38-46$ & \multirow[t]{6}{*}{1} & \multirow[t]{6}{*}{ Semi } \\
\hline & & (E)- $\beta$-farnesene & & $256-561$ & & $16-20$ & & \\
\hline & & $(Z, Z) \alpha$-farnesene & & $103-250$ & & $6-10$ & & \\
\hline & & germacrene-D & & $208-441$ & & $13-15$ & & \\
\hline & & $\beta$-bisabolene & & $179-436$ & & $11-14$ & & \\
\hline & & unknown compound & & $77-189$ & & $4-7$ & & \\
\hline \multirow[t]{3}{*}{$\begin{array}{l}\text { Arey et al., } \\
1995\end{array}$} & $\begin{array}{c}\text { Black Sage (Salvia } \\
\text { mellifera) }\end{array}$ & $\begin{array}{l}\alpha \text {-cubebene } \\
\alpha \text {-copaene } \\
\beta \text {-bourbonene } \\
\beta \text {-Car } \\
\text { germacrene-D }\end{array}$ & $\left\{\begin{array}{l}<20-5600 \\
\text { field } \\
286-2272 \\
\text { greenhouse }(\mathrm{gh})\end{array}\right.$ & & & $\begin{array}{l}<1-70 \\
\text { field } \\
1.3-7.1 \\
(\mathrm{gh}) \\
\end{array}$ & \multirow[t]{3}{*}{2} & \multirow[t]{3}{*}{ Yes } \\
\hline & Greenbark & Not stated & & & & & & \\
\hline & $\begin{array}{l}\text { California } \\
\text { Sagebrush }\end{array}$ & Not stated & & & & & & \\
\hline \multirow{2}{*}{$\begin{array}{l}\text { Arimura et al., } \\
2004\end{array}$} & \multirow{2}{*}{$\begin{array}{l}\text { Hybrid Poplar } \\
\text { (Populus } \\
\text { trichocarpa } \mathbf{x} \\
\text { deltoides) }\end{array}$} & germacrene-D & 80 (a), 10 (b), 0 (c) & & & & \multirow[t]{2}{*}{3} & \multirow[t]{2}{*}{ Yes } \\
\hline & & $(E, E)$ - $\alpha$-farnesene & 60 (a), 30 (b), 0 (c) & & & & & \\
\hline \multirow{6}{*}{$\begin{array}{l}\text { Chen et al., } \\
2003\end{array}$} & \multirow{6}{*}{$\begin{array}{c}\text { Thale Crest } \\
\text { (Arabidopsis } \\
\text { thaliana, Columbia } \\
\text { ecotype) }\end{array}$} & $\beta$-Car & & $\sim 11.5$ & & $\sim 44$ & \multirow[t]{6}{*}{4} & \multirow[t]{6}{*}{ Semi } \\
\hline & & thujopsene & & $\sim 1.25$ & & $\sim 5$ & & \\
\hline & & $\alpha$-humulene & & $\sim 0.9$ & & $\sim 3$ & & \\
\hline & & $\beta$-chamigrene & & $\sim 0.75$ & & $\sim 3$ & & \\
\hline & & $\beta$-farnesene & & $\sim 0.6$ & & $\sim 2$ & & \\
\hline & & cuparene & & $\sim 0.25$ & & $\sim 1$ & & \\
\hline \multirow[t]{3}{*}{$\begin{array}{l}\text { Ciccioli et al., } \\
1999\end{array}$} & \multirow{3}{*}{$\begin{array}{c}\text { Varieties of } \\
\text { Citrus sinensis and } \\
\text { Citrus Clementi } \\
\text { sampled during } \\
\text { July }\end{array}$} & $\begin{array}{l}\beta-\text { Car } \\
\text { (Valencia Navel) }\end{array}$ & & & $\sim 36000$ & $50-70$ & \multirow[t]{3}{*}{5} & \multirow[t]{3}{*}{ Yes } \\
\hline & & $\begin{array}{l}\beta-\text { Car (Valencia Navel-late } \\
\text { and Clemenule) }\end{array}$ & & & $\begin{array}{l}180000- \\
360000\end{array}$ & $50-70$ & & \\
\hline & & $\alpha$-humulene & & & & $<1$ & & \\
\hline \multirow{4}{*}{$\begin{array}{l}\text { Degenhardt and } \\
\text { Lincoln, } 2006\end{array}$} & Marsh Elder & germacrene-D & & & $9200-15000$ & $\sim 6$ & 6 & Yes \\
\hline & (Iva frutescens) & $\beta-$ Car & & & $1000-11000$ & $1-4$ & & \\
\hline & & $\alpha$-humulene & & & $1000-2000$ & $\sim 1$ & & \\
\hline & & cis- $\beta$-guaiene & & & $0-8000$ & $0-5$ & & \\
\hline $\begin{array}{l}\text { De Moraes et } \\
\text { al., } 2001\end{array}$ & $\begin{array}{l}\text { Tobacco } \\
\text { (Nicotiana }\end{array}$ & $\beta-$ Car & & $\begin{array}{l}3741 \text { (a) } \\
1227 \text { (b) }\end{array}$ & & $\begin{array}{l}\sim 46.6 \text { (a) } \\
\sim 23.5 \text { (b) }\end{array}$ & 7 & Semi \\
\hline & tabacum) & $\alpha$-farnesene & & $\begin{array}{l}610(a) \\
445(b)\end{array}$ & & $\begin{array}{l}\sim 7.6 \text { (a) } \\
\sim 8.5 \text { (b) }\end{array}$ & & \\
\hline & & $\alpha$-humulene & & $\begin{array}{l}111(a) \\
42(b)\end{array}$ & & $\begin{array}{l}\sim 1.4 \text { (a) } \\
\sim 0.8 \text { (b) }\end{array}$ & & \\
\hline & & unidentified SQT & & $\begin{array}{l}574(\mathrm{a}) \\
481(\mathrm{~b})\end{array}$ & & $\begin{array}{l}\sim 7.2 \text { (a) } \\
\sim 9.2 \text { (b) }\end{array}$ & & \\
\hline $\begin{array}{l}\text { Dudareva et al., } \\
2005\end{array}$ & $\begin{array}{c}\text { Snapdragon } \\
\text { flowers } \\
\text { (Antirrhinum } \\
\text { majus cv. Maryland } \\
\text { True Pink) } \\
\end{array}$ & nerolidol & $\sim 200-4000^{* * *}$ & & & $\sim 45-65$ & 8 & Semi \\
\hline Gouinguené and & Corn & $\beta-$ Car & Widely-varying emission & & & $0.7-15$ & 9 & No \\
\hline Turlings, 2002 & (Zea mays) & $\alpha$-bergamotene & rates for all compounds; & & & $7.5-40$ & & \\
\hline & & $\beta$-farnesene & none explicitly stated & & & $0-40$ & & \\
\hline & & $\begin{array}{l}\alpha \text {-farnesene and/or } \\
\beta \text {-bisabolene }\end{array}$ & & & & $0.5-2.8$ & & \\
\hline & & (E)-nerolidol & & & & $0-2.5$ & & \\
\hline $\begin{array}{l}\text { Hakola et al., } \\
2001\end{array}$ & $\begin{array}{c}\text { Downy Birch } \\
\text { (Betula pubescens) }\end{array}$ & $\begin{array}{l}\text { cis-caryophyllene } \\
\beta \text {-Car } \\
\alpha \text {-farnesene }\end{array}$ & $310-6940$ & & & $<5-\sim 75$ & 10 & Yes \\
\hline $\begin{array}{l}\text { Hakola et al., } \\
2006\end{array}$ & $\begin{array}{c}\text { Scots Pine } \\
\text { (Pinus sylvestris } \\
\text { L.) }\end{array}$ & $\begin{array}{l}\beta-\text { Car } \\
\text { Other SQT }\end{array}$ & $\begin{array}{l}\text { 11(a), 171(b), 42(c), } \\
\text { 15(d) } \\
1(a), 39(b), 19(c), 7(d)\end{array}$ & & & $\begin{array}{l}0-26 \\
0-6\end{array}$ & 11 & Yes \\
\hline
\end{tabular}


Table 2. Continued.

\begin{tabular}{|c|c|c|c|c|c|c|c|}
\hline \multirow{2}{*}{$\begin{array}{l}\text { Hansen and } \\
\text { Seufert, } 1999\end{array}$} & \multirow{2}{*}{$\begin{array}{l}\text { Citrus sinensis } \\
\text { (L.) OSBECK }\end{array}$} & $\beta-\operatorname{Car}$ (normal conditions) & 410 & & $40-45$ & \multirow[t]{2}{*}{12} & \multirow[t]{2}{*}{ Yes } \\
\hline & & $\beta-$ Car (varying water stress) & $20-410$ & & & & \\
\hline \multirow{18}{*}{$\begin{array}{l}\text { Helmig et al., } \\
1999\end{array}$} & Ironwood & Total SQT & 800 & & 14.8 & \multirow[t]{18}{*}{13} & \multirow[t]{18}{*}{ Semi } \\
\hline & Post Oak & Total SQT & 78000 & & 48.8 & & \\
\hline & Black Oak & Total SQT & 8500 & & 11.2 & & \\
\hline & White Oak & Total SQT & 2600 & & 3.2 & & \\
\hline & Speckled Alder & Total SQT & 300 & & 1.8 & & \\
\hline & Black Cherry & Total SQT & 3800 & & 34.5 & & \\
\hline & Red Raspberry & Total SQT & 73000 & & 35.3 & & \\
\hline & White spruce & Total SQT & 1500 & & 2.5 & & \\
\hline & Eastern Hemlock & Total SQT & 100 & & 0.9 & & \\
\hline & Labrador Tea & Total SQT & 14000 & & 21.5 & & \\
\hline & Subalpine Fir & Total SQT & 800 & & 5.0 & & \\
\hline & Aspen & Total SQT & 300 & & 1.1 & & \\
\hline & Big Sagebrush & Total SQT & 1300 & & 4.2 & & \\
\hline & Englemann Spruce & Total SQT & $<100$ & & $<2.4$ & & \\
\hline & Lodgepole Pine & Total SQT & 300 & & 0.6 & & \\
\hline & Gambel Oak & Total SQT & 1500 & & 2.2 & & \\
\hline & Rabbit Brush & Total SQT & 3200 & & 2.9 & & \\
\hline & Salt Bush & Total SQT & 15000 & & 31.3 & & \\
\hline \multirow{4}{*}{$\begin{array}{l}\text { Helmig et al., } \\
2006\end{array}$} & \multirow{4}{*}{$\begin{array}{l}\text { Loblolly Pine } \\
\text { (Pinus taeda L.) }\end{array}$} & $\beta-\mathrm{Car}$ & 297 & & \multirow{4}{*}{$\int \sim 50$} & \multirow[t]{4}{*}{14} & \multirow[t]{4}{*}{ Yes } \\
\hline & & $\alpha$-bergamotene & 54 & & & & \\
\hline & & $\alpha$-humulene & 45 & & & & \\
\hline & & $\beta$-farnesene & 50 & & & & \\
\hline \multirow[t]{8}{*}{$\begin{array}{l}\text { Helmig et al., } \\
2007\end{array}$} & $\begin{array}{l}\text { Gray Pine (Pinus } \\
\text { sabiniana) }\end{array}$ & Total SQT & 60 (greenhouse) & & 1.5 & \multirow[t]{8}{*}{15} & \multirow[t]{8}{*}{ Yes } \\
\hline & $\begin{array}{l}\text { Scots Pine (Pinus } \\
\text { sylvestris) }\end{array}$ & Total SQT & $<4$ (greenhouse) & & 0.0 & & \\
\hline & $\begin{array}{l}\text { Ponderosa Pine } \\
\text { (Pinus ponderos) }\end{array}$ & Total SQT & 70 (July and August) & & 12.3 & & \\
\hline & $\begin{array}{c}\text { Shortleaf Pine } \\
\text { (Pinus echinata) }\end{array}$ & Total SQT & 90 (September) & & 37.5 & & \\
\hline & $\begin{array}{l}\text { Beach Pine (Pinus } \\
\text { contorta) }\end{array}$ & Total SQT & 100 (September) & & 37.0 & & \\
\hline & $\begin{array}{l}\text { White Pine (Pinus } \\
\text { strobus) }\end{array}$ & Total SQT & 100 (June, July) & & 11.9 & & \\
\hline & $\begin{array}{l}\text { Red Pine (Pinus } \\
\text { resinosa) }\end{array}$ & Total SQT & 80 (July) & & 11.6 & & \\
\hline & $\begin{array}{l}\text { Loblolly Pine } \\
\text { (Pinus taeda) }\end{array}$ & Total SQT & $\begin{array}{l}150, \quad 350 \quad \text { (August, } \\
\text { September) }\end{array}$ & & $46.9,42.7$ & & \\
\hline \multirow{2}{*}{$\begin{array}{l}\text { Helsper et al., } \\
1998\end{array}$} & \multirow{2}{*}{$\begin{array}{l}\text { Rosa hybrida L. } \\
\text { cv. Honesty } \\
\text { (flowers) }\end{array}$} & $\beta-$ Car & & 100 & 0.4 & \multirow[t]{2}{*}{16} & \multirow[t]{2}{*}{ Semi } \\
\hline & & $\beta$-cubebene & & 350 & 1.4 & & \\
\hline \multirow{8}{*}{$\begin{array}{l}\text { Holzke et al., } \\
2006\end{array}$} & Scots Pine & $\gamma$-cadinene & $0.64,5.94,0.93,0.02$ & & & 17 & Yes \\
\hline & (Pinus sylvestris & $\delta$-cadinene & $0.31,5.26,1.24,0.01$ & & & & \\
\hline & L.) & $\alpha$-muurolene & $0.20,3.83,0.68,<0.01$ & & & & \\
\hline & & Aromadendrene & $0.17,<0.01,0.16,<0.01$ & & & & \\
\hline & & $\beta$-Car & $0.66,26.20,1.66,0.01$ & & & & \\
\hline & & $\beta$-cedrene & $0.46,31.23,5.49,0.07$ & & & & \\
\hline & & $\beta$-bourbonene & $0.14,0.49,0.05,<0.01$ & & & & \\
\hline & & $\alpha$-ylangene & $0.16,0.31,0.14,<0.01$ & & & & \\
\hline $\begin{array}{l}\text { König, et al., } \\
1995\end{array}$ & $\begin{array}{c}\text { Hornbeam } \\
\text { (Carpinus betulus) }\end{array}$ & $\beta-$ Car & 20.3 & & 12.6 & 18 & Yes \\
\hline & $\begin{array}{c}\text { Birch (Betula } \\
\text { pendula) }\end{array}$ & other SQT & 61.2 & & 11.0 & & \\
\hline Martin et al., & Norway Spruce & $\alpha$-bergamotene & (a) $0.0^{*}$ (b) $10.5^{*}$ & & $0,0.2$ & 19 & Yes \\
\hline 2003 & (Picea abies L. & $\beta$-farnesene & (a) $26.7^{*}$ (b) $2653.4^{*}$ & & $4.3,55.1$ & & \\
\hline & Karst) & $\alpha$-farnesene & (a) $29.4^{*}$, (b) $46.8^{*}$ & & $4.7,1.0$ & & \\
\hline & & $\alpha$-bisabolene & (a) $62.9 *$ (b) $972.3 *$ & & $10.1,20.2$ & & \\
\hline & & other SQT & (a) $0.0^{*}$ (b) $10.5^{*}$ & & $0,0.2$ & & \\
\hline $\begin{array}{l}\text { Ormeño et al., } \\
2007 \mathrm{a}\end{array}$ & $\begin{array}{l}\text { Rosemary } \\
\text { (Rosmarinus } \\
\text { officinalis L.) }\end{array}$ & $\begin{array}{l}\alpha \text {-humulene } \\
\alpha \text {-muurolene } \\
2 \text { unidentified SQT } \\
\beta \text {-Car }\end{array}$ & $\{\sim 300-400$ & & $5-15$ & 20 & Semi \\
\hline & $\begin{array}{c}\text { Aleppo Pine } \\
\text { (Pinus halepensis } \\
\text { Mill.) }\end{array}$ & $\begin{array}{l}\alpha \text {-humulene } \\
\alpha \text {-muurolene } \\
\beta \text {-bourbonene } \\
\text { copaene } \\
\beta \text {-Car } \\
2 \text { unidentified SQT }\end{array}$ & $\sim 200-500$ & & $6-23$ & & \\
\hline
\end{tabular}


Table 2. Continued.

\begin{tabular}{|c|c|c|c|c|c|c|}
\hline $\begin{array}{l}\text { Ormeño et al., } \\
\text { 2007a (cont.) }\end{array}$ & $\begin{array}{c}\text { Rock Rose } \\
\text { (Cistus albidus L.) }\end{array}$ & $\begin{array}{l}\text { AR-curcumene } \\
\alpha \text {-zingiberene } \\
\beta \text {-bourbonene } \\
\beta \text {-Car } \\
\alpha \text {-humulene } \\
\text { allo-aromadendrene } \\
\text { copaene } \\
\alpha \text {-muurolene } \\
2 \text { unidentified SQT } \\
\end{array}$ & $\sim 2200-3000$ & $\sim 76$ & & \\
\hline \multirow{18}{*}{$\begin{array}{l}\text { Ormeño et al., } \\
\text { 2007b }\end{array}$} & \multirow{4}{*}{$\begin{array}{l}\text { Rosemary } \\
\text { (Rosmarinus } \\
\text { officinalis L.) }\end{array}$} & allo-aromadendrene & $\sim 405$ & 5.8 & \multirow[t]{18}{*}{21} & \multirow[t]{18}{*}{ Yes } \\
\hline & & germacrene-d & $\sim 475$ & 6.8 & & \\
\hline & & $\alpha$-zingiberene & $\sim 500$ & 7.13 & & \\
\hline & & d-cadinene & $\sim 525$ & 7.5 & & \\
\hline & \multirow{4}{*}{$\begin{array}{c}\text { Aleppo Pine } \\
\text { (Pinus halepensis } \\
\text { Mill.) }\end{array}$} & $\alpha$-humulene & $\sim 20$ & 5.3 & & \\
\hline & & allo-aromadendrene & $\sim 35$ & 9.8 & & \\
\hline & & germacrene-d & $\sim 20$ & 6.5 & & \\
\hline & & d-cadinene & 25 & 7.7 & & \\
\hline & \multirow{7}{*}{$\begin{array}{c}\text { Rock Rose } \\
\text { (Cistus albidus L.) }\end{array}$} & $\beta$-bourbonene & $\sim 155$ & 7.0 & & \\
\hline & & $\beta$-Car & $\sim 150$ & 6.8 & & \\
\hline & & $\alpha$-humulene & $\sim 15$ & 0.7 & & \\
\hline & & allo-aromadendrene & $\sim 150$ & 6.7 & & \\
\hline & & germacrene-d & $\sim 145$ & 6.6 & & \\
\hline & & AR-curcumene & $\sim 235$ & 10.7 & & \\
\hline & & $\alpha$-zingiberene & $\sim 245$ & 11.1 & & \\
\hline & \multirow{3}{*}{$\begin{array}{c}\text { Kermes Oak } \\
\text { (Quercus } \\
\text { coccifera L.) }\end{array}$} & \multirow{3}{*}{$\begin{array}{l}\text { allo-aromadendrene } \\
\text { germacrene-d } \\
\text { d-cadinene }\end{array}$} & $\sim 85$ & 5.4 & & \\
\hline & & & $\sim 70$ & 4.5 & & \\
\hline & & & $\sim 140$ & 8.9 & & \\
\hline \multirow{6}{*}{$\begin{array}{l}\text { Ruther and } \\
\text { Kleier, } 2005\end{array}$} & \multirow{6}{*}{$\begin{array}{c}\text { Corn } \\
\text { (Zea mays cv. } \\
\text { Delprim) }\end{array}$} & (E)- $\beta$-farnesene & $0.25 * * *$ & 6 & \multirow[t]{6}{*}{22} & \multirow[t]{6}{*}{ Semi } \\
\hline & & sesquithujene & $0.2^{* * *}$ & 5 & & \\
\hline & & $\beta-\mathrm{Car}$ & $0.1125^{* * *}$ & 3 & & \\
\hline & & (E)- $\alpha$-bergamotene & $0.0875^{* * *}$ & 2 & & \\
\hline & & $\beta$-bisabolene & $0.0125 * * *$ & $<1$ & & \\
\hline & & 7-epi-sesquithujene & $0.0125^{* * *}$ & $<1$ & & \\
\hline \multirow[t]{2}{*}{$\begin{array}{l}\text { Ruuskanen et } \\
\text { al., } 2007\end{array}$} & \multirow[t]{2}{*}{$\begin{array}{l}\text { Siberian Larch } \\
\text { (Larix sibirica) }\end{array}$} & $\alpha$-farnesene & ) & $\begin{array}{l}10 \text { (a) } \\
\sim 1 \text { (b) }\end{array}$ & \multirow[t]{2}{*}{23} & \multirow[t]{2}{*}{ Yes } \\
\hline & & $\begin{array}{l}\beta \text {-Car } \\
\text { longifolene } \\
\beta \text {-cubebene } \\
\text { s-cadinene } \\
\alpha \text {-humulene } \\
\beta \text {-bourbonene } \\
\text { iso-longifolene }\end{array}$ & $\left\{\begin{array}{l}0.958-1.798(a) \\
0.404-0.596(b)\end{array}\right.$ & $\begin{array}{l}\sim 2 \text { (a) } \\
\sim 2 \text { (b) }\end{array}$ & & \\
\hline \multirow{2}{*}{$\begin{array}{l}\text { Schuh et al., } \\
1997\end{array}$} & \multirow{2}{*}{$\begin{array}{c}\text { Sunflower } \\
\text { (Helianthus } \\
\text { annuus L. cv. } \\
\text { giganteus) }\end{array}$} & $\beta-\mathrm{Car}$ & $93-303$ & $\sim 26$ & \multirow[t]{2}{*}{24} & \multirow[t]{2}{*}{ Yes } \\
\hline & & $\alpha$-humulene & $9.9-38$ & $\sim 3$ & & \\
\hline \multirow[t]{2}{*}{$\begin{array}{l}\text { Tarvainen et al., } \\
2005\end{array}$} & \multirow[t]{2}{*}{$\begin{array}{l}\text { Scots Pine (Pinus } \\
\text { sylvestris L.) }\end{array}$} & $\beta-\mathrm{Car}$ & $<4.5-37,160-533,0-158$ & $\begin{array}{ll}0-2, & 3-26, \\
0-4 & \end{array}$ & \multirow[t]{2}{*}{25} & \multirow[t]{2}{*}{ Yes } \\
\hline & & unidentified SQT & & & & \\
\hline \multirow{6}{*}{$\begin{array}{l}\text { Vuorinen et al., } \\
2005\end{array}$} & & $\alpha-$ copaene (July) & $278-1498^{* *}$ & $\sim 5-8$ & 26 & Yes \\
\hline & (Betula pendula & $\alpha$-copaene (August) & $10-65^{* *}$ & $\sim 2-3$ & & \\
\hline & & $\beta-$ Car (July) & $224-1184 * *$ & $\sim 3-4$ & & \\
\hline & & $\beta-$ Car (August) & $4-54^{* *}$ & $\sim 1-2$ & & \\
\hline & & $\alpha$-farnesene (July) & $91-991 * *$ & $\sim 2-11$ & & \\
\hline & & $\alpha$-farnesene (August) & $13-326 * *$ & $\sim 1-12$ & & \\
\hline Zhang et al., & European Birch & Total SQT & $25-211,12-60$ & $\sim 20,11$ & 27 & Semi \\
\hline 1999 & Downy Birch & Total SQT & 663-1494, 204-363 & $\sim 22,9$ & & \\
\hline & Trembling Aspen & Total SQT & $132-632,0-11$ & $\sim 41,19$ & & \\
\hline & Black Elder & Total SQT & $2-62,1-13$ & $\sim 22,17$ & & \\
\hline
\end{tabular}

to sample collection to allow initial bursts of emissions to subside. Many of the early published SQT ER studies may have reported artificially elevated ERs caused from the rough handling of vegetation specimens and premature sample collection. Observed emissions changes due to artificial disturbances in enclosure experiments suggest that emissions bursts might also be caused in nature by natural disturbances (e.g. violent weather events), but this potential effect has yet to be characterized and published.

\subsubsection{Infestation}

Among the known BVOC chemical species, many SQT play critical roles in plant-insect and plant-plant interactions. For instance, Turlings et al. (1995) studied the release of SQT and other BVOC by corn seedlings as a response to caterpillar feeding, and demonstrated that their emissions attracted wasps which parasitize caterpillars. In another study, tobacco plants were shown to emit herbivore-induced BVOC (including SQT), which repel ovipositing female moths, thus 
Table 2. Footnotes.

* ERs were published as $n g \mathrm{~g}_{\text {freshweight }}^{-1} \mathrm{~h}^{-1}$. For coniferous tree species, the ratio of fresh to dry weight is $\sim 1.5-2.5$. Therefore, the ERs listed may be a factor of 1.5-2.5 lower than what would have been reported if dry weight had been used.

** ERs were published as $\mathrm{ng} \mathrm{g}_{\text {freshweight }}^{-1} \mathrm{~h}^{-1}$. For deciduous tree species, the ratio of fresh to dry weight is $\sim 2-3$. Therefore, the ERs given in this study may be a factor of 2-3 lower than what would have been reported if dry weight had been used.

*** ERs were published as $\mathrm{ng} \mathrm{g}_{\text {freshweight }}^{-1} \mathrm{~h}^{-1}$. For non-tree vegetation, the ratio of fresh to dry weight can vary considerably. Therefore, the ERs reported here cannot be quantitatively scaled to $\operatorname{ng~}_{D W}^{-1} \mathrm{~h}^{-1}$ without fresh:dry weight ratio data for the plant specimens used in this study.

Remarks: This information is presented as: Experimental conditions, sample dates, \# of specimens measured, \# of replicates per specimen. In cases where experiments were performed in a laboratory or greenhouse, the word "lab" is given instead of listing sample dates. For all items, "n.s." appears if this information was not stated.

1. ERs represent mean emissions ranges from whole plants. Samples were collected at temperatures between $16-19^{\circ} \mathrm{C}$ and at an illuminance of $1500-1600 \mathrm{~lm} \mathrm{~m}^{-2}$. These ERs were not normalized for biomass, though plants within each group were similar in weight, lab, $\sim 84,1$.

2. Emissions were normalized to $30^{\circ} \mathrm{C}$. Large volume flow-through plant enclosure chamber with high air flow ( $\left.\sim 40 \mathrm{LPM}\right)$ of charcoalscrubbed medical-grade air (ambient [CO2]). Whole plants were enclosed. Enclosures were allowed 20-30 mins of equilibration time. For California Sagebrush, substantial variability in emission profiles was observed between individual plants; the authors felt that disturbances of glandular trichomes on leaf surfaces may have influenced these emissions, for Black Sage in field: April-October, 7, 6-21, for Black Sage in greenhouse: lab, 3, 5-6.

3. Trees were maintained in a greenhouse under summer conditions (daytime temperatures: $18.3-21.8^{\circ} \mathrm{C}$; relative humidity: $24.5-47.2 \%$ ). ERs are given for (a) FTC-infested trees after $24 \mathrm{~h}$ of feeding, (b) trees damaged by mechanical wounding, and (c) undamaged control trees. All emissions were recorded from directly-damaged leaves (non-systemic leaves), lab, 5, 1.

4. 6-week-old whole plants were sampled at $23^{\circ} \mathrm{C}$ and $150 \mu \mathrm{mol} \mathrm{m}^{-2} \mathrm{~s}^{-1}$ PAR using dynamic enclosures purged with purified air. 8-h samples were collected, and listed ERs represent hourly averages per whole plant, lab, 5, 4.

5. ERs were normalized to $30^{\circ} \mathrm{C}$ and $1000 \mu \mathrm{mol} \mathrm{m}^{-2} \mathrm{~s}^{-1} \mathrm{PPFD}$. Temperatures within the enclosures were maintained within $2-3^{\circ} \mathrm{C}$ of ambient temperatures. Ozone was reduced to $<10 \%$ of ambient levels, July, C. sinensis: 5 , n.s.; C. clementi: 1 , n.s.

6. Volatiles were collected at $22^{\circ} \mathrm{C}$. Enclosure was purged with compressed medical-grade air, lab, 12, 1-10.

7. Average emission rates given for approximately (a) noon, $50 \mathrm{~h}$ after initial herbivore damage, and (b) 08:00 p.m., $58 \mathrm{~h}$ after damage. 8 -week-old plants were sampled at $29 \pm 4^{\circ} \mathrm{C}$, lab, n.s., n.s.

8. Experiments were conducted using cut 5-d-old flowers. ERs reflect the range observed across light $\left(150 \mu \mathrm{mol} \mathrm{m}^{-2} \mathrm{~s}^{-1} \mathrm{PAR}\right)$ and dark periods under a constant air temperature of $21^{\circ} \mathrm{C}, 1 \mathrm{ab}, 3,2-3$.

9. ERs depended on abiotic factor being tested and whether or not plant was damaged, lab, $>60,1-12$.

10. ERs were standardized to $30^{\circ} \mathrm{C}$. Air was scrubbed of ozone (using $\mathrm{MnO}_{2}$-coated copper nets) before being flown into enclosure, n.s., 18 , n.s.

11. ERs were standardized to $30^{\circ} \mathrm{C}$ and are calculated as averages from sunlit branches for (a) April-May, (b) June-July, (c) August, and (d) September-October. Enclosure inlet air was ozone-scrubbed, April-October, 1, 2.

12. ER associated with non-drought conditions for cuvette air temperatures of $30^{\circ} \mathrm{C}$ and PPFD of $1069 \mu \mathrm{mol} \mathrm{m}^{-2} \mathrm{~s}^{-1}$. Water stress ERs depended on level of stress, cuvette air temperatures were $29-31^{\circ} \mathrm{C}$ at PPFDs of $1016-1095 \mu \mathrm{mol} \mathrm{m}^{-2} \mathrm{~s}^{-1}$. Purge air was scrubbed, lab, 1, 1-20.

13. Emission rates were normalized to $30^{\circ} \mathrm{C}$ using response curves developed from MT. Hydrocarbon-free air was flown into enclosures. 63 vegetation species from 3 distinct sites (each investigated during a different period of the summer) in the US were screened for BVOC emissions. Healthy, sunlit branches were cut from trees and samples were collected after the enclosures had been allowed to equilibrate for $10 \mathrm{~min}$. Most of the emission data obtained during this experiment were collected under relatively constant light and temperature values around $40^{\circ} \mathrm{C}$ and $1700 \mu \mathrm{mol} \mathrm{m}^{-2} \mathrm{~s}^{-1}$ PPFD. $\sim 25$ separate SQT were observed, though individual SQT were not speciated in this study. Errors were stated to have been within a factor of 2-3 based on adverse enclosure conditions due to higher-than-ambient temperatures inside of enclosures, possible disturbances to branches, and analytical uncertainties, June-August, 1-4, 1.

14. ER was normalized to $30^{\circ} \mathrm{C}$. Individual SQT temperature-dependent emissions curves were also presented. Enclosures were allowed to equilibrate for $\sim 1 \mathrm{~d}$ prior to sampling. Purge air was scrubbed. Needle temperatures were recorded. Uncertainty in SQT ER was stated as $\pm 15 \%$, Late September, 4, 10-34.

15. Total SQT ER represents BER, normalized to $30^{\circ} \mathrm{C}$. SQT emissions were found in 7 of 8 pine species screened. Measurements were conducted during different months depending on species. Enclosures were allowed to equilibrate for $\sim 0.5-1 \mathrm{~d}$ prior to sampling. Purge air was scrubbed. Needle temperatures were recorded, March-September, 1-4, 1-34. 
Table 2. Footnotes continued.

16. Temperature was kept constant at $20^{\circ} \mathrm{C}$, with a relative humidity of $75 \%$ and a constant PPFD of $210 \mu \mathrm{mol} \mathrm{m}^{-2} \mathrm{~s}^{-1}$. Petal temperature was monitored by attaching a thermocouple to outer petals of flowers, lab, 3, 1 .

17. ERs were normalized to $30^{\circ} \mathrm{C}$. The four numbers reported for each compound represent average daytime emissions for March-Apr., May, June-July, and Aug.-Oct. Nighttime measurements showed non-detectable emission levels. Enclosure air was VOC and $\mathrm{O}_{3}$ scrubbed. Enclosures equilibrated overnight prior to sample collection, March-October, 2, 50.

18. Of nine agricultural and natural species studied, only two were found to be SQT emitters; Hornbeam and Birch. Aside from $\beta$-Car, other SQT were not speciated. Enclosure temperatures were $\sim 23^{\circ} \mathrm{C}$. Ambient air was charcoal-scrubbed, sampling began 20 mins after plants were enclosed, minimal disturbance to plants was emphasized, Mid-May, 1 (per species), 1-2.

19. All experiments were conducted at a constant temperature of $22^{\circ} \mathrm{C}, 75 \%$ relative humidity, and at a PPFD of $350 \mu \mathrm{mol} \mathrm{m}{ }^{-2} \mathrm{~s}^{-1}$. SQT ERs (and SQT \% of total BVOC emissions) reported for both (a) untreated control plants and (b) plants $\sim 24 \mathrm{~h}$ after being treated with methyl jasmonate, lab, 3, 1 .

20. Samples were collected between 11:00-15:30 (solar time). ERs listed are non-standardized means and represent ranges observed across plants growing in siliceous and calcareous soils. Sun- and shade-exposed twigs were sampled. Ambient temperatures during sampling were $22-25^{\circ} \mathrm{C}$ and PAR ranged from 750-960 $\mu \mathrm{mol} \mathrm{m}^{-2} \mathrm{~s}^{-1}$. Enclosures were semi-dynamic and were set up $\sim 10 \mathrm{~min}$ prior to sampling. Inlet air was pollution-free, late March, 24 (per species), 1.

21. Healthy sun- and shade-exposed twigs were sampled. ERs given in Table 2 reflect those of unstressed plants and were standardized to $30^{\circ} \mathrm{C}$. Ambient temperature was $\sim 32^{\circ} \mathrm{C}$ (enclosure temperatures were not reported), PAR was $\sim 1310 \mu \mathrm{mol} \mathrm{m}^{-2} \mathrm{~s}^{-1}$. Enclosures were set up and purged with zero air beginning 15 min prior to sampling, early June, 6 (per species), 1.

22. Enclosure air was charcoal-scrubbed. Volatiles were collected for an 8 -hour period; the ERs listed in Table 2 were divided by 8 to give average ERs per hour, lab, 9, 1 .

23. Sun-exposed branches were sampled. Listed ERs were standardized to $30^{\circ} \mathrm{C}$ for samples collected from (a) late May $\rightarrow$ early August and (b) late August $\rightarrow$ late September, though stated ranges also reflect standardized ERs obtained using varying values of $\beta$. Enclosure temperatures ranged from $5 \rightarrow 40^{\circ} \mathrm{C}$ and PAR varied from $0 \rightarrow \geq 1400 \mu \mathrm{mol} \mathrm{m}^{-2} \mathrm{~s}^{-1}$. Enclosures were set up $\sim 4 \mathrm{~h}$ prior to sampling and inlet air was ozone-scrubbed, May $\rightarrow$ September, 1, 60-120.

24. Samples were collected at a PPFD of $820 \mu \mathrm{mol} \mathrm{m} \mathrm{m}^{-2} \mathrm{~s}^{-1}$ and at leaf temperatures between 25 and $26^{\circ} \mathrm{C}$. Leaf temperatures inside the enclosure were stringently monitored and controlled using microthermistors. At constant temperatures and light intensities, variability in emissions for an individual plant ranged from 15 to $30 \%$ over a one d period, and within a factor of two over the course of one week. There was a significant diurnal variation, with lower nighttime emissions, even though temperatures at night were within $3^{\circ} \mathrm{C}$ of daytime temperatures. Enclosure air was scrubbed of oxidants and VOC. Enclosure was tested for surface losses of the analytes; the authors stated that emission rates calculated for most analytes could be up to $20 \%$ understated, lab, 11, n.s.

25. ERs listed represent samples collected in spring, early summer, and late summer $\rightarrow$ autumn, respectively and were normalized to $30^{\circ} \mathrm{C}$ and PPFD of $1000 \mu \mathrm{mol} \mathrm{m}{ }^{-2} \mathrm{~s}^{-1} . \mathrm{O}_{3}$ was removed from inlet air; enclosures were allowed to equilibrate from $\sim \mathrm{several} \mathrm{h}$ to $>1 \mathrm{~d}$ before samples were collected, spring $\rightarrow$ autumn, 2, 22-132.

26. ERs represent ranges detected from 2 control clones (i.e., not exposed to elevated $\mathrm{CO}_{2}$ or $\mathrm{O}_{3}$ ) during June and Aug. (listed as: June range, August range). Enclosure air was scrubbed. Samples were collected at $22-25^{\circ} \mathrm{C}$ from detached twigs, June, August, 8,1 (each month).

27. ERs are ranges detected during June and Aug. (listed as: June range, Aug. range, for both ERs and \% SQT contribution to total BVOC). Bag temperatures ranged from $16.4-39.9^{\circ} \mathrm{C}$. Numerous SQT, non-identified SQT and SQT alcohols were detected. Blank bag testing indicated no detectable release of volatiles, nor were ambient biogenic BVOC observed in inlet air, June, August, 2 (per species), 2-3 (per month).

thwarting future damage from larval feeding (De Moraes et al., 2001). These studies imply that SQT emissions may be significantly enhanced from disturbances such as insect herbivory. Many recent studies have characterized induced BVOC emissions. Several representative reports are described below.

In Black Sage, SQT made up approximately $4 \%$ of total BVOC emissions for plants grown in a greenhouse, and $<1-$ $70 \%$ for plants growing in the field, many of which were infested with aphids (Arey et al., 1995). In late February through April, plants growing in the field emitted a much higher percentage of SQT than plants measured in the greenhouse (from late November to early February). The percentage of SQT (of total BVOC emissions) in field plants was especially high during March and April, when most plants had not yet bloomed and some were heavily infested with aphids. The investigators also noted that field plants emitted significantly less total BVOC and were growing under hotter and more arid conditions than their greenhouse counterparts, possibly leading to smaller available terpene pools in these plants. 
Table 3. Contribution of individual SQT chemical species to observed SQT emissions by plant species (in \%).

\begin{tabular}{|c|c|c|c|c|c|c|c|c|c|c|c|c|c|c|c|c|c|c|}
\hline Plant species & 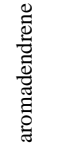 & 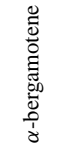 & $\begin{array}{l}\stackrel{0}{0} \\
\frac{0}{0} \\
\frac{0}{0} \\
\frac{0}{0} \\
0 \\
0\end{array}$ & 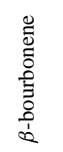 & 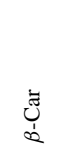 & 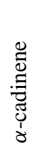 & 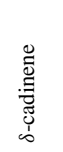 & 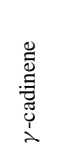 & 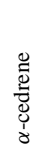 & 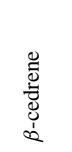 & $\begin{array}{l}\overline{0} \\
\text { t⿹ } \\
\text { d }\end{array}$ & 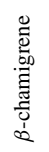 & $\begin{array}{l}\tilde{D} \\
\tilde{\tilde{J}} \\
\tilde{Z} \\
\dot{i} \\
\dot{1}\end{array}$ & 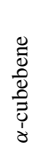 & 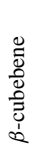 & 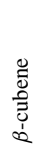 & 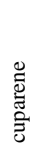 & 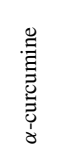 \\
\hline Potato & & & $11-14$ & & $38-46$ & & & & & & & & & & & & & \\
\hline Thale Cress & & & & & $\sim 75$ & & & & & & & $\sim 5$ & & & & & $<2$ & \\
\hline Citrus & & & & & $>98$ & & & & & & & & & & & & & \\
\hline Marsh Elder & & & & & $5-39$ & & & & & & & & & & & & & \\
\hline \multicolumn{19}{|l|}{ Snapdragon flowers } \\
\hline Corn & & $24-71$ & & & $1-17$ & & & & & & & & & & & & & \\
\hline Loblolly Pine & & 12 & & & 66 & & & & & & & & & & & & & \\
\hline Gray Pine & & & & 1 & & & & & & & 3 & & 1 & & & & & \\
\hline Ponderosa Pine & & & & & 22 & & 6 & 7 & & & & & & 4 & & & & \\
\hline Shortleaf Pine & & 100 & & & & & & & & & & & & & & & & \\
\hline Beach Pine & & 77 & & & 6 & & 5 & & & & & & & & & & & \\
\hline White Pine & & & & & 7 & & 2 & & 1 & & & & & & 4 & & & \\
\hline Red Pine & & & & & & & & 1 & & & & & & & 4 & & & \\
\hline Loblolly Pine & & $9-13$ & & & $26-67$ & & & $0-2$ & $0-1$ & & $0-1$ & & & & & & & \\
\hline Rose flowers & & & & & 22 & & & & & & & & & & 78 & & & \\
\hline Scots Pine & $0-6$ & & & $0-5$ & $9-36$ & & $7-12$ & $8-23$ & & $17-64$ & & & & & & & & \\
\hline Hornbeam & & & & & 100 & & & & & & & & & & & & & \\
\hline Norway Spruce & & & 53 & & & & & & & & & & & & & & & \\
\hline Aleppo Pine & 33 & & & & & & 26 & & & & & & & & & & & \\
\hline Kermes Oak & 29 & & & & & & 47 & & & & & & & & & & & \\
\hline Rock Rose & 14 & & & 14 & 14 & & & & & & & & & & & & & 22 \\
\hline Rosemary & 21 & & & & & & 28 & & & & & & & & & & & \\
\hline Corn & & 13 & 2 & & 17 & & & & & & & & & & & & & \\
\hline \multicolumn{19}{|l|}{ Siberian Larch } \\
\hline Sunflower & & & & & $\sim 91$ & & & & & & & & & & & & & \\
\hline Silver Birch & & & & & $10-47$ & & & & & & & & $15-46$ & & & & & \\
\hline European Birch & $3-5$ & & & $0-27$ & $17-25$ & $0-5$ & & & & & & & $0-21$ & & & $0-7$ & & $0-2$ \\
\hline Downy Birch & $4-6$ & & & $0-2$ & $46-61$ & $0-2$ & & & & & & & $4-14$ & $0-1$ & & $0-6$ & & \\
\hline Trembling Aspen & $2-13$ & & & $0-1$ & $0-15$ & & & & & & & & $0-5$ & $0-1$ & & $0-3$ & & $18-51$ \\
\hline Black Elder & & & & $0-3$ & $0-5$ & & & & & & & & & & & & & $5-27$ \\
\hline
\end{tabular}

\begin{tabular}{|c|c|c|c|c|c|c|c|c|c|c|c|c|c|c|c|c|}
\hline Plant species & $\begin{array}{l}\text { D } \\
\stackrel{0}{0} \\
\frac{0}{0} \\
0 \\
0\end{array}$ & 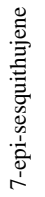 & 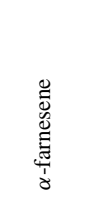 & 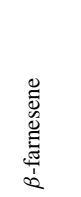 & 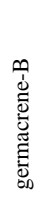 & 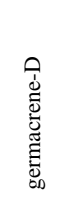 & 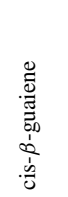 & 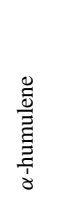 & 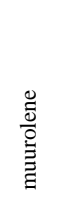 & $\begin{array}{l}\bar{o} \\
\frac{0}{0} \\
\stackrel{0}{0} \\
\stackrel{0}{0}\end{array}$ & 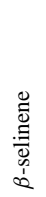 & 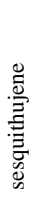 & $\begin{array}{l}\stackrel{0}{0} \\
\stackrel{0}{0} \\
\stackrel{0}{\Xi} \\
\stackrel{\Xi}{\Xi}\end{array}$ & 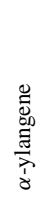 & 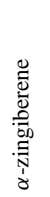 & Reference \\
\hline Potato & & & $6-10$ & $16-20$ & & $13-15$ & & & & & & & & & & Agelopoulos et al. (2000) \\
\hline Thale Cress & & & & $\sim 4$ & & & & $\sim 6$ & & & & & $\sim 8$ & & & Chen et al. (2003) \\
\hline Citrus & & & & & & & & $<2$ & & & & & & & & Ciccioli et al. (1999) \\
\hline Marsh Elder & & & & & & $48-54$ & $0-42$ & $5-7$ & & & & & & & & Degenhardt and Lincoln (2006) \\
\hline Snapdragon flowers & & & & & & & & & & 100 & & & & & & Dudareva et al. (2005) \\
\hline Corn & & & $1-10$ & $0-70$ & & & & & & $0-4$ & & & & & & Gouinguené and Turlings (2002) \\
\hline Loblolly Pine & & & & 11 & & & & 10 & & & & & & & & Helmig et al. (2006) \\
\hline Gray Pine & & & 20 & 77 & & & & & & & & & & & & Helmig et al. (2007) \\
\hline Ponderosa Pine & & & & 27 & & 10 & & 10 & 10 & & & & & & & Helmig et al. (2007) \\
\hline Shortleaf Pine & & & & & & & & & & & & & & & & Helmig et al. (2007) \\
\hline Beach Pine & & & 4 & 3 & & 5 & & & 1 & & & & & & & Helmig et al. (2007) \\
\hline White Pine & & & 57 & 3 & & & & 5 & 13 & & 8 & & & & & Helmig et al. (2007) \\
\hline Red Pine & & & 55 & 41 & & & & & & & 3 & & & & & Helmig et al. (2007) \\
\hline Loblolly Pine & $0-1$ & & $0-1$ & $8-17$ & $0-1$ & & & $8-13$ & $0-27$ & $0-6$ & & & & & & Helmig et al. (2007) \\
\hline Rose flowers & & & & & & & & & & & & & & & & Helsper et al. (1998) \\
\hline Scots Pine & & & & & & & & & $0-7$ & & & & & $0-6$ & & Holzke et al. (2006) \\
\hline Hornbeam & & & & & & & & & & & & & & & & König et al. (1995) \\
\hline Norway Spruce & & & 25 & 22 & & & & & & & & & & & & Martin et al. (2003) \\
\hline Aleppo Pine & & & & & & 22 & & 18 & & & & & & & & Ormeño et al. (2007b) \\
\hline Kermes Oak & & & & & & 24 & & & & & & & & & & Ormeño et al. (2007b) \\
\hline Rock Rose & & & & & & 13 & & 1 & & & & & & & 22 & Ormeño et al. (2007b) \\
\hline Rosemary & & & & & & 25 & & & & & & & & & 26 & Ormeño et al. (2007b) \\
\hline Corn & & 2 & & 37 & & & & & & & & 30 & & & & Ruther and Kleier (2005) \\
\hline Siberian Larch & & & $\sim 33-83$ & & & & & & & & & & & & & Ruuskanen et al. (2007) \\
\hline Sunflower & & & & & & & & $\sim 9$ & & & & & & & & Schuh et al. (1997) \\
\hline Silver Birch & & & $25-75$ & & & & & & & & & & & & & Vuorinen et al. (2005) \\
\hline European Birch & & & $5-19$ & & & & & & $3-49$ & & & & & & & Zhang et al. (1999) \\
\hline Downy Birch & & & $0-4$ & & & & & $\sim 7$ & $0-1$ & & & & & & & Zhang et al. (1999) \\
\hline Trembling Aspen & & & $10-33$ & & & & & $3-36$ & $0-2$ & & & & & & & Zhang et al. (1999) \\
\hline Black Elder & & & $33-40$ & & & & & & & & & & & & & Zhang et al. (1999) \\
\hline
\end{tabular}


Tomato shoots subjected to caterpillar herbivory emitted elevated levels of SQT (relative to undamaged plants) immediately following insect damage (Maes and Debergh, 2003). Both MT and SQT emissions in the damaged plants exhibited a fluctuating day and nighttime pattern, with peaks observed in both light and dark phases (though nighttime peaks were smaller than those seen during the photoperiod), suggesting that the herbivory occurred both during the day and at night. When the caterpillars were removed from the plants, emissions declined to normal (undamaged) levels within hours. The authors concluded that induced emissions were the result of volatile escape from trichomes ruptured during feeding.

Methyl jasmonate (MeJA) was applied to Norway Spruce saplings to induce chemical defense responses while avoiding physical damage to plant tissues (Martin et al., 2003). The authors observed that induced SQT emissions in saplings followed a diurnal time course, peaking during light periods. SQT emissions increased by a factor of 30 , and became more dominant in the mixture of volatiles emitted. Maximum emissions occurred during the photoperiod 1-2 days after treatment, and then slowly declined. The authors noted that terpenes were largely absent from needle oleoresin, and were the dominant products of terpene synthase activity observed post-treatment. These observations led to the conclusion that the induced volatiles appeared to have been synthesized de novo following MeJA treatment, in lieu of being released from storage pools.

The emissions and molecular regulation of volatiles released by forest tent caterpillar (FTC)-infested poplar leaves and non-infested systemic leaves (from infested trees) were examined by Arimura et al. (2004). Both infested and systemic leaves released similar blends of volatiles, including SQT, though directly infested leaves released higher overall amounts. FTC-induced volatile emissions were low on the first day after FTC feeding, but became significant within 24-h, lasting for $\sim 3$ days during feeding. After removal of FTC, emissions of germacrene-D continued for $24 \mathrm{~h}$ before declining. FTC-infested leaves emitted higher amounts of germacrene-D than mechanically-wounded leaves. Unwounded control trees did not produce detectable amounts of analytes. The amount of germacrene-D released from FTC-infested trees was variable, possibly due to tree-to-tree variations and/or FTC feeding behavior variations. Emissions of other induced volatiles followed a pattern similar to that of germacrene-D. Based on the isolation and study of a germacrene-D synthase during this investigation, the authors concluded that transient and diurnal volatile emissions are at least partly regulated by the level of transcription of this synthase.

In the 1999 Ciccioli et al. study, investigators noted that some of the Navel orange trees sampled for emissions were infected with the Citrus quick decline virus, but these trees did not show significantly different emission rates than healthy trees. This is an interesting finding, suggesting the possibility that emissions might only be induced by infesta- tions that are potentially alleviated through chemical signaling of herbivore predators, and not necessarily by diseases such as viral infections.

\subsubsection{Variability among individuals}

The emissions from individuals within a species can vary considerably, even when other environmental variables are constant. For sunflower, Schuh et al. (1997) observed variability in SQT emission rates on the order of a factor of $\sim 3-4$ between individual plants, even though all individuals were at the same vegetative stage and light/temperature regimes were identical. A similar phenomenon was observed in Scots Pine, with near-simultaneous measurements from 2 trees revealing differences (in total SQT ERs) from a few percent to $\sim$ one order of magnitude depending on the month considered (Holzke et al., 2006). Additionally, the contributions of individual SQT chemical species to total SQT emissions varied considerably between individual Scots Pine trees. Significant intraspecific variability has also been reported for Downy Birch (Hakola et al., 2001) and potato (Agelopoulos et al., 2000). Not surprisingly, variability of up to one order of magnitude has been observed across different cultivated varieties of Citrus (Ciccioli et al., 1999) as well as between ecotypes of Arabidopsis thaliana (Tholl et al., 2005). Though the reasons for such substantial variability between individuals are not well understood, numerous factors may be at work, such as prior herbivore feeding, which can possibly trigger permanent biochemical changes in terpenoid pathways and rates of terpene synthase gene expression (Huber et al., 2004). In any case, this significant source of variation in ERs should be kept in mind when applying results from emissions experiments.

\section{Sesquiterpene emitting plant species, quantities of emissions, and chemical species identifications}

\subsection{Sesquiterpene emission rates}

Table 2 outlines 27 studies that report biogenic SQT emissions. Also presented are the plant species studied, the quantities and chemical species of SQT emitted, and (where available) the percent contributions of those compounds to total BVOC emissions for each plant species. For each study there is a brief assessment of whether or not the emissions data are quantitative. This determination was made based on several factors, such as whether or not the emissions were normalized to plant biomass, whether or not there was substantial uncertainty regarding quantification of emissions (as determined by the authors of each published study), etc. Also included for each study is a footnote containing ancillary information (i.e., sampling dates, number of specimens measured, etc.) that may be of use to the reader. Our efforts to present reported emissions data in a standardized fashion were limited in some cases, as discussed below. 
Many, but not all, of the studies summarized in Table 2 report emission rates normalized to a standard temperature. Additional information regarding temperature data and other information associated with reported ERs is provided in the Table 2 footnotes. Some investigators have reported SQT ERs normalized to plant biomass, while others have not. These ERs have been separated into appropriate columns. While most investigators who normalized emissions to plant biomass used dry weight as a metric, a few reported volatiles released per quantity of fresh weight. The ERs in Table 2 that fall into this latter category have been marked with asterisks, and suggested dry to fresh weight conversions are given following Table 2, for both coniferous and broadleaf tree species. These suggested conversions are based on personal observations made by the authors (unpublished data). Still other studies reported ERs per $\mathrm{m}^{2}$ leaf area, and the reader will find these listed in a separate column.

From an inspection of Table 2 it is obvious that there exists substantial variability in emissions between and within plant species, with ER values observed from $<10$ to $78000 \mathrm{ng} \mathrm{g}_{\text {DW }}^{-1} \mathrm{~h}^{-1}$. The percent contribution of SQT to total observed BVOC also varies considerably, ranging from $<1$ to $100 \%$ of the total BVOC ER. When one considers only the emissions data from Table 2 that were reported as emissions per gram (fresh or dry weight) of plant matter per hour, the average SQT ER across all species is $\sim 3000 \mathrm{ng} \mathrm{g}_{\mathrm{DW}}^{-1} \mathrm{~h}^{-1}$. It is important to recognize that these values may not reflect average "real-world" emissions. Numerous ERs reported in Table 2 are based on single measurements (using one plant specimen), and many of the early SQT enclosure studies did not carefully control enclosure temperatures and other important experimental parameters, such as disturbances to the enclosed specimens during enclosure set-up. These studies may have reported artificially-enhanced SQT emissions, while other studies may have neglected some of the analytical steps necessary to quantitatively measure SQT, thereby possibly underestimating SQT fluxes. It should also be noted that the vegetation species chosen in these experiments most likely do not represent a random selection, but instead are frequently-sampled SQT-emitting species chosen because of researcher interest in studying SQT emissions. Therefore, these values should not be considered as "average" emissions from all terrestrial vegetation. We attempted to calculate average SQT ERs as a function of plant functional type (PFT) using all available data (with the exception of published ERs which exceeded $50000 \mathrm{ng} \mathrm{g}_{\text {DW }}^{-1} \mathrm{~h}^{-1}$, as these were possibly elevated due to disturbance-induced emissions bursts). When normalized to $30^{\circ} \mathrm{C}$, average values (presented with corresponding standard deviations) for coniferous trees, broadleaf trees, shrubs, and crops are $290 \pm 410,1410 \pm 2200$, $7060 \pm 6830$ and $190 \pm 430 \mathrm{ng} \mathrm{g}_{\text {DW }}^{-1} \mathrm{~h}^{-1}$, respectively. Given the paucity of available data and the high standard deviations of PFT-specific ERs, SQT emissions currently cannot be accurately characterized according to plant growth forms.
3.2 Relative abundances of reported sesquiterpene compounds

It is important to consider and evaluate which SQT chemical species are the important players in biogenic emissions, as individual compounds have significantly different reactivities, and thus different atmospheric fates.

In order to determine which biogenically-emitted SQT have been observed to dominate individual SQT emission profiles, we examined the relative contribution of individual SQT chemical species to total observed SQT emissions for different plant species, based on studies that have speciated these compounds. Table 3 contains a list of individual SQT and their relative contribution to overall SQT emissions from these studies. As disturbance is known to affect the quality of SQT blends emitted, investigations that reported disturbance-induced SQT emissions were excluded from Table 3. Unidentified SQT chemical species were also excluded, although numerous investigators have reported ERs for these compounds. $\beta$-Car is the most frequently reported SQT, and is also one of the most abundant SQT chemical species within many SQT emissions profiles. $\alpha$ and $\beta$-farnesene are also prominent contributors to observed SQT profiles, as is $\alpha$-humulene. Though less frequently reported, aromadendrene, $\alpha$-bergamotene, $\delta$-cadinene, and germacrene-D have all been observed to contribute significantly to biogenic SQT emissions in some species.

\section{Conclusions}

The task of synthesizing the various published SQT emissions studies is greatly complicated by a lack of standardized reporting practices. This reality creates a major difficulty for modelers of biogenic SQT processes, who seek to increase both vegetation and chemical species information included in current biogenic emissions inventories. Some authors normalize SQT emissions to dry vegetation weight, while others use fresh weight. Many authors standardize their emission factors according to previously-existing algorithms, while others report values which represent emissions across a range of temperatures. And while most authors report light intensity in terms of PPFD $\left(\mu \mathrm{mol} \mathrm{m}^{-2} \mathrm{~s}^{-1}\right)$, some report this parameter as illuminance (e.g., $1 \mathrm{~m} \mathrm{~m}^{-2} \mathrm{~s}^{-1}$ ), which is a poorlysuited measure of light intensity within the context of plant science. One particularly useful improvement to reporting practices is to include the values of empirical parameters used to calculate standardized emission rates, such as $\beta$ factors and other coefficients. Additionally, specific temperatures and PPFDs measured during sample acquisition periods should be made available, rather than ranges of values observed over multiple sample collection periods. Finally, when standardizing emissions to biomass, it would be sensible to report emissions as a function of dry vegetation weight, 
in lieu of using fresh weight as a metric, since leaf water content can vary significantly even within a single specimen.

$\beta$-Car, $\alpha$ - and $\beta$-farnesene, and $\alpha$-humulene are the most commonly reported biogenically-emitted SQT chemical species, though aromadendrene, $\alpha$-bergamotene, $\delta$ cadinene, and germacrene-D have also been identified as significant compounds in some plant species. SQT emissions typically increase with temperature. The effects of light and stomatal control on SQT emissions are less clear. Significant seasonal variation observed in the contribution of SQT to total BVOC emissions creates the possibility that SQT and their roles in atmospheric processes are especially prominent during warmer times of the year, particularly in late spring to mid-summer. Further characterization of seasonally-dependant SQT emissions is needed. Another source of substantial uncertainty is the variability in SQT emissions among individuals within a species, and (to an even greater extent) between different species. Though the enclosure studies clearly show that disturbances to vegetation are important in controlling short-term SQT emissions bursts, the time-averaged magnitude of disturbance-induced emissions (from herbivores and severe weather events) under ambient conditions is highly uncertain, and additional studies of disturbance as a driver of SQT emissions are needed.

Based on the biogenic SQT emissions studies reviewed here, SQT emission rates cover a wide range of values, varying between individuals and across species, as well as at different temperatures and phenological states. These published emission rates range from $<10$ to $>10000 \mathrm{ng} \mathrm{g}_{\mathrm{DW}}^{-1} \mathrm{~h}^{-1}$, but this range is heavily influenced by early SQT studies, which may have reported artificially-elevated SQT emission rates due to higher-than-ambient enclosure temperatures and disturbances to enclosed vegetation prior to and during sample collection. Furthermore, vegetation species that were chosen for these experiments probably do not represent a random selection, but instead are species that were selected because of researcher interest in studying SQT emissions. Therefore, averages that are computed based on published data probably result in higher SQT emissions than what is actually emitted from the biosphere. These observations make the characterization of landscape and global SQT fluxes highly uncertain, and underscore the need for screening of numerous species as well as the collection of multiple replicates from several individuals within a given species. It is still unclear whether SQT emissions may be adequately modeled as a function of plant functional type (e.g. broadleaf trees, herbs, etc.) or according to some other grouping scheme. An appropriate approach for quantifying global distributions of SQT emissions remains to be elucidated.

Acknowledgements. The National Center for Atmospheric Research is supported by the National Science Foundation (NSF). T. R. Duhl received a fellowship from the NSF-sponsored Biosphere Atmosphere Research and Training (BART) - Graduate Education Research and Training (IGERT) program administered by Western Michigan University. Part of this research was also funded by NSF grant ATM \#0304704 and EPA grant RD83107901-0. Any opinions, findings and conclusions expressed in this material are those of the authors and do not necessarily reflect the views of the funding agencies.

Edited by: A. Neftel

\section{References}

Agelopoulos, N. G., Chamberlain, K., and Pickett, J. A.: Factors affecting volatile emissions of intact Potato plants, Solanum tuberosum: variability of quantities and stability of ratios, J. Chem. Ecol., 26, 497-511, 2000.

Arey, J., Crowley, D. E., Crowley, M., Resketo, M., and Lester, J.: Hydrocarbon emissions from natural vegetation in California's South Coast Air Basin, Atmos. Environ., 29, 2977-2988, 1995.

Arimura, G., Huber, D. P. W., and Bohlmann, J.: Forest tent caterpillars (Malacosoma disstria) induce local and systemic diurnal emissions of terpenoid volatiles in hybrid poplar (Populus trichocarpa $\times$ deltoides): cDNA cloning, functional characterization, and patterns of gene expression of (-)-germacrene D synthase, PtdTPS1, The Plant J., 37, 603-616, 2004.

Atkinson, R. and Arey, J.: Gas-phase tropospheric chemistry of biogenic volatile organic compounds: a review, Atmos. Environ., 37(Supp. No. 2), S197-S219, 2003.

Chen, F., Tholl, D., D’Auria, J.C., Farooq, A., Pichersky, E., and Gershenzon, J.: Biosynthesis and emission of terpenoid volatiles from Arabidopsis flowers, Plant Cell, 15, 481-494, 2003.

Ciccioli, P., Brancaleoni, E., Frattoni, M., Di Palo, V., Valentini, R., Tirone, G., Seufert, G., Bertin, N., Hansen, U., Csiky, O., Lenz, R., and Sharma, M.: Emission of reactive terpene compounds from orange orchards and their removal by within-canopy processes, J. Geophys. Res., 104, 8077-8094, 1999.

Degenhardt, D. C. and Lincoln, D. E.: Volatile emissions from an odorous plant in response to herbivory and methyl jasmonate exposure, J. Chem. Ecol., 32, 725-743, 2006.

Dement, W. A., Tyson, B. J., and Mooney, H. A.: Mechanism of monoterpene volatilization in Salvia mellifera, Phytochemistry, 14, 2555-2557, 1975.

De Moraes, C. M., Mescher, M. C., and Tumlinson, J. H.: Caterpillar-induced nocturnal plant volatiles repel conspecific females, Nature, 410, 577-580, 2001.

Dudareva, N., Andersson, S., Orlova, I., Gatto, N., Reichelt, M., Rhodes, D., Boland, W., and Gershenzon, J.: The nonmevalonate pathway supports both monoterpene and sesquiterpene formation in snapdragon flowers, Proc. Natl. Acad. Sci., 102, 933-938, 2005.

Fehsenfeld, F., Calvert, J., Fall, R., Goldan, P., Guenther, A. B., Hewitt, C. N., Lamb, B., Liu, S., Trainer, M., Westberg, H., and Zimmerman, P.: Emissions of volatile organic compounds from vegetation and the implications for atmospheric chemistry, Global Biogeochem. Cycles, 6, 389-430, 1992.

Fuentes, J. D., Lerdau, M., Atkinson, R., Baldocchi, D., Bottenheim, J. W., Ciccioli, P., Lamb, B., Geron, C., Gu, L., Guenther, A., Sharkey, T. D., and Stockwell, W.: Biogenic hydrocarbons in the atmospheric boundary layer : a review, Bull. Amer. Met. Soc. $81,1537-1575,2000$. 
Geron, C., Guenther, A., Greenberg, J., Karl, T., and Rasmussen, R.: Biogenic volatile organic compound emissions from desert vegetation of the southwestern US, Atmos. Environ., 40, 16451660,2006

Gouinguené, S. P. and Turlings, T. C. J.: The effects of abiotic factors on induced volatile emissions in corn plants, Plant Phys., 129, 1296-1307, 2002.

Guenther, A. B., Zimmerman, P. R., Harley, P. C., Monson, R. K., and Fall, R.: Isoprene and monoterpene emission rate variability: model evaluations and sensitivity analyses, J. Geophys. Res., 98, 12 609-12 617, 1993.

Guenther, A., Zimmerman, P., and Wildermuth, M.: Natural volatile organic compound emission rate estimates for U.S. woodland landscapes, Atmos. Environ., 28, 1197-1210, 1994.

Hakola, H., Laurila, T., Lindfors, V., Hellén, H., Gaman, A., and Rinne, J.: Variation of the VOC emission rates of birch species during the growing season, Boreal Environ. Res., 6, 237-249, 2001.

Hakola, H., Tarvainen, V., Bäck, J., Ranta, H., Bonn, B., Rinne, J., and Kulmala, M.: Seasonal variation of mono- and sesquiterpene emission rates of Scots Pine, Biogeosciences, 3, 93-101, 2006, http://www.biogeosciences.net/3/93/2006/.

Hansen, U. and Seufert, G.: Terpenoid emission from Citrus sinensis (L.) OSBECK under drought stress, Phys. Chem. Earth (B), 24, 681-687, 1999.

Hansen, U. and Seufert, G.: Temperature and light dependence of $\beta$-caryophyllene emission rates, J. Geophys. Res., 108, 4801, doi:10.1029/2003JD003853, 2003.

Harley, P., Guenther, A., and Zimmerman, P.: Effects of light, temperature and canopy position on net photosynthesis and isoprene emission from sweetgum (Liquidambar styraciflua) leaves, Tree Physiology, 16, 25-32, 1996.

Helmig, D., Klinger, L. F., Guenther, A., Vierling, L., Geron, C., and Zimmerman, P.: Biogenic volatile organic compound emissions (BVOCs): I. Identifications from three continental sites in the U.S., Chemosphere, 38, 2163-2187, 1999.

Helmig, D., Revermann, T., Pollmann, J., Kaltschmidt, O., Jiménez Hernández, A., Bocquet, F., and David, D.: Calibration system and analytical considerations for quantitative sesquiterpene measurements in air, J. Chrom. A, 1002, 193-211, 2003.

Helmig, D., Bocquet, F., Pollmann, J., and Revermann, T.: Analytical techniques for sesquiterpene emission rate studies in vegetation enclosure experiments, Atmos. Environ., 38, 557-572, 2004.

Helmig, D., Ortega, J., Guenther, A., Herrick, J., and Geron, C.: Sesquiterpene emissions from Loblolly Pine and their potential contribution to biogenic aerosol formation in the Southeastern US, Atmos. Environ., 40, 4150-4157, 2006.

Helmig, D., Ortega, J., Duhl, T., Tanner, D., Guenther, A., Harley, P., Wiedinmyer, C., Milford, J., and Sakulyanontvittaya, T.: Sesquiterpene emissions from pine trees - Identifications, emission rates, and flux estimates for the contiguous United States, Environ. Sci. Technol. 41, 1545-1553, 2007.

Helsper, J. P. F. G., Davies, J. A., Bouwmeester, H. J., Krol, A. F., and van Kampen, M. H.: Circadian rhythmicity in emission of volatile compounds by flowers of Rosa hybrida L. cv. Honesty, Planta, 207, 88-95, 1998.

Holzke, C., Hoffmann, T., Jaeger, L., Koppmann, R., and Zimmer, W.: Diurnal and seasonal variation of monoterpene and sesquiterpene emissions from Scots Pine (Pinus silvestris L.), Atmos. Environ., 40, 3174-3185, 2006.

Huber, D. P. W., Ralph, S., and Bohlmann, J.: Genomic hardwiring and phenotypic plasticity of terpenoid-based defenses in conifers, J. Chem. Ecol., 30, 2399-2418, 2004.

Kesselmeier, J. and Staudt, M.: Biogenic volatile organic compounds (VOC): an overview on emission, physiology, and ecology, J. Atmos. Chem., 33, 23-88, 1999.

Kesselmeier, J., Ciccioli, P., Kuhn, U., Stefani, P., Biesenthal, T., Rottenberger, S., Wolf, A., Vitullo, M., Valentini, R., Nobre, A., Kabat, P., and Andreae, M. O.: Volatile organic compound emissions in relation to plant carbon fixation and the terrestrial carbon budget, Global Biogeochem. Cycles, 16, 1126 , doi:10.1029/2001GB001813, 2002.

Komenda, M., Parusel, E., Wedel, A., and Koppmann, R.: Measurements of biogenic VOC emissions: sampling, analysis and calibration, Atmos. Environ., 35, 2069-2080, 2001.

König, G., Brunda, M., Puxbaum, H., Hewitt, C. N., Duckham, S. C., and Rudolph, J.: Relative contribution of oxygenated hydrocarbons to the total biogenic VOC emissions of selected midEuropean agricultural and natural plant species, Atmos. Environ., 29, 861-874, 1995.

Kulmala, M., Hari, P., Laaksonen, A., Vesala, T., and Viisanen, Y.: Research unit of physics, chemistry and biology of atmospheric composition and climate change: overview of recent results, Boreal Environ. Res., 10, 459-477, 2005.

Lathière, J., Hauglustaine, D. A., Friend, A. D., De NobletDucoudrè, N., Viovy, N., and Folberth, G. A.: Impact of climate variability and land use changes on global biogenic volatile organic compound emissions, Atmos. Chem. Phys., 6, 2129-2146, 2006, http://www.atmos-chem-phys.net/6/2129/2006/.

Maes, K. and Debergh, P. C.: Volatiles emitted from in vitro grown tomato shoots during abiotic and biotic stress, Plant Cell Tissue and Organ Culture, 75, 73-78, 2003.

Martin, D. M., Gershenzon, J., and Bohlmann, J.: Induction of volatile terpene biosynthesis and diurnal emission by methyl jasmonate in foliage of Norway spruce, Plant Phys., 132, 15861599, 2003.

Merfort, I.: Review of the analytical techniques for sesquiterpenes and sesquiterpene lactones, J. Chromatogr. A, 967, 115-130, 2002.

Ormeño, E., Fernandez, C., Bousquet-Mélou, A., Greff, S., Morin, E., Robles, C., Vila, B., and Bonin, G.: Monoterpene and sesquiterpene emissions of three Mediterranean species through calcareous and siliceous soils in natural conditions, Atmos. Environ., 41, 629-639, 2007a.

Ormeño, E., Mévy, J.P., Vila, B., Bousquet-Mélou, A., Greff, S., Bonin, G., and Fernandez, C.: Water deficit stress induces different monoterpene and sesquiterpene emission changes in Mediterranean species. Relationship between terpene emissions and plant water potential, Chemosphere, 67, 276-284, 2007b.

Pollmann, J., Ortega, J., and Helmig, D.: Analysis of atmospheric sesquiterpenes: Sampling losses and mitigation of ozone interferences, Environ. Sci. Technol., 39, 9620-9629, 2005.

Ruther, J. and Kleier, S.: Plant-plant signaling: Ethylene synergizes volatile emission in Zea mays induced by exposure to (Z)3-Hexen-1-ol, J. Chem. Ecol., 31, 2217-2222, 2005.

Ruuskanen, T. M., Hakola, H., Kajos, M. K., Hellén, H., Tarvainen, 
V., and Rinne, J.: Volatile organic compound emissions from Siberian larch, Atmos. Environ., 41, 5807-5812, 2007.

Schade G. W. and Goldstein, A. H.: Fluxes of oxygenated volatile organic compounds from a ponderosa pine plantation, J. Geophys. Res., 106, 3111-3123, 2001.

Schuh, G., Heiden, A. C., Hoffmann, Th., Kahl, J., Rockel, P., Rudolph, J., and Wildt, J.: Emissions of volatile organic compounds from Sunflower and Beech: dependence on temperature and light intensity, J. Atmos. Chem., 27, 291-318, 1997.

Tarvainen, V., Hakola, H., Hellén, H., Bäck, J., Hari, P., and Kulmala, M.: Temperature and light dependence of the VOC emissions of Scots Pine, Atmos. Chem. Phys., 5, 989-998, 2005, http://www.atmos-chem-phys.net/5/989/2005/.

Tholl, D., Chen, F., Petri, J., Gershenzon, J., and Pichersky, E.: Two sesquiterpene synthases are responsible for the complex mixture of sesquiterpenes emitted from Arabidopsis flowers, Plant J., 42, 757-771, 2005.

Tholl, D., Boland, W., Hansel, A., Loreto, F., Röse, U.S.R., and Schnitzler, J.-P.: Practical approaches to plant volatile analysis, Plant J., 45, 540-560, 2006.
Turlings, T. C. J., Loughrin, J. H., McCall, P. J., Röse, U. S. R., Lewis, W. J., and Tumlinson, J. H.: How caterpillar-damaged plants protect themselves by attracting parasitic wasps, Proc. Natl. Acad. Sci., 92, 4169-4174, 1995.

Vuorinen, T., Nerg, A.-M., Ibrahim, M. A., Reddy, G. V. P., and Holopainen, J. K.: Emission of Plutella xylostella-induced compounds from cabbages grown at elevated $\mathrm{CO}_{2}$ and orientation behavior of the natural enemies, Plant Phys., 135, 1984-1992, 2004.

Vuorinen, T., Nerg, A.-M., Vapaavuori, E., and Holopainen, J. K.: Emission of volatile organic compounds from two silver birch (Betula pendula Roth) clones grown under ambient and elevated $\mathrm{CO}_{2}$ and different $\mathrm{O}_{3}$ concentrations, Atmos. Environ., 39, 1185-1197, 2005.

Zhang, Q.-H., Birgersson, G., Zhu, J., Löfstedt, C., Löfqvist, J., and Schlyter, F.: Leaf volatiles from nonhost deciduous trees: variation by tree species, season, and temperature, and electrophysiological activity in Ips typographus, J. Chem. Ecol., 25, 19231943, 1999. 